\title{
Evaluation of Supervised machine learning classifiers for detecting the degrees of possibility of Coronavirus disease infection
}

\author{
Lebanese University \\ Elie Yammine \\ Lebanese University

\section{Walid Fahs} \\ Islamic University of Lebanon

\section{Rida Khatoun} \\ Telecom ParisTech
}

Abbas Rammal ( $\nabla$ rammal_abbass@hotmail.com )

\section{Research Article}

Keywords: Machine learning, Supervised learning algorithms, Classification, Coronavirus disease, Model prediction, Support Vector Machine, Naïve Bayesian Classifier, Decision Tree Classifier, Linear Discriminant Analysis, Artificial Neural Network, Extended Gamma, Associative Classifier Naive Associative Classifier

Posted Date: March 7th, 2022

DOI: https://doi.org/10.21203/rs.3.rs-958969/v1

License: (9) This work is licensed under a Creative Commons Attribution 4.0 International License. Read Full License 


\section{Evaluation of Supervised machine learning classifiers for detecting the}

2

3

4

5

6

7

8

9

10

11

12

13

14

15

16

17

18

19

20

21

22

23

24

25

26

27 2

\section{degrees of possibility of Coronavirus disease infection}

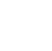

${ }^{1}$ Islamic University of Lebanon, Faculty of Engineering, 30014, Wardenieh, Lebanon.

${ }^{2}$ Lebanese University, Faculty of Science, Statistics and Informatics department, Beirut, Lebanon.

${ }^{3}$ Lebanese University, Faculty of Information, Data Science department, Beirut, Lebanon.

${ }^{4}$ Telecom ParisTech, Paris, France

(1)

1 rammal_abbass@hotmail.com, e.yammine@st.ul.edu.lb,walid.fahs@iul.edu.lb, rida.khatoun@telecom-paris.fr

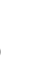

4

5

6

7

8

9


29 Correspondence to Abbas Rammal

30 First author: Abbas Rammal, Ph.D., Assistant professor, Lebanese University, Faculty of 31 Science, Statistics and computer sciences department, Beirut, Lebanon, 32 abbas.rammal@ul.edu.lb

33 Second author: Elie Yammine, Master's degree in applied statistics, Lebanese University, 34 Faculty of Science, computer science and statistics department, Beirut, Lebanon, 35 e.yammine@st.ul.edu.lb

36 Third author: Walid Fahs, Phd, Assistant professor, Islamic University of Lebanon, Faculty 37 of Engineering, transportation, and civil engineering department, Wardenieh, Lebanon, 38 walid.fahs@iul.edu.lb

39 Fourth author: Rida Khatoun, Ph.D., Assistant professor, Telecom ParisTech, Paris, France, 40 rida.khatoun@telecom-paris.fr 
The COVID-19 virus spread from China throughout the world, causing out of control challenges to the public health community. During the epidemic, it is difficult to act against infectious disease due to its unknown trends, so the spread prediction becomes difficult in light of the scarce data. With the absence of treatment for COVID-19 infection, countries have taken some mitigation steps and policies, such as general lockdown and social distance measures, but there has been variation in the extent of viral spread due to several additional factors. Prediction methods based on Artificial intelligence (AI) and Machine Learning (ML) can help in suggesting new policies or even assessing the effectiveness of the existing ones. Such methods have attracted wide attention from researchers implementing statistical modeling and machine learning methods.

The objective of this study is to examine different supervised classification approaches to detect the degrees of possibility of Coronavirus disease infection in different countries. Naïve Bayesian Classifier (NBC), Decision Tree Classifier (DTC), Linear Discriminant Analysis (LDA), Support Vector Machine (SVM) and Artificial Neural Network classification (ANN) are machine learning algorithms used for the prediction of coronavirus disease cases according to the time of their evolution while considering data collected from official reports and scientific journals. Since we collected mixed data, we suggested to also apply the supervised classifiers available for mixed data, such as Extended Gamma (EG) and Naive Associative Classifier (NAC).

The results showed that ANN and DTC supervised algorithms allow better discrimination between the degrees of possibility of Coronavirus disease infection among advanced methods like NBC, SVM, NAC, EG and LDA. 

Model prediction, Support Vector Machine, Naïve Bayesian Classifier, Decision Tree Associative Classifier Naive Associative Classifier.

82

83

84

85

86

87

88

89

90

91

92

93

94

95

96

97

98

99

100

101

102

103

104

105

106

107

108

109

3. Introduction 
111 In December 2019, the novel coronavirus appeared in Wuhan city in China [1] as the first case

112 was reported to the World Health Organization (W.H.O) on 31 December 2019. The virus

113 created a global threat and was announced as coronavirus disease (COVID-19) by the W.H.O

114 on 11 February 2020. The COVID-19 epidemic caused serious global socio-economic turmoil

115 [2]. As of 29 July 2020, more than 17 million cases were recorded. The virus was detected in

116215 countries causing more than 665,000 deaths [3].

118 The early detection of any disease, whether it is infectious or not, is a critical task for rapid

119 treatment to save more lives [4]. Fast diagnosis and screening processes help prevent the spread

120 of pandemic diseases like SARS-CoV-2 [5]. Moreover, predicting the probability degree of

121 getting infected with covid-19 may help national authorities in taking proactive mitigation

122 measures to contain possible epidemic waves of infections. The biggest challenge is to build a

123 technique to improve the detection of covid-19 disease. Several Data Science and Artificial

124 Intelligence techniques have been used to aid the Coronavirus Response [6,7]. In the literature

125 review, deep learning methods to predict the structure of proteins and their interactions with

126 chemical compounds to facilitate new antiviral drugs/vaccines or recommend current drugs is

127 widely used [8]. A machine learning-based prognostic model to predict if a patient infected

128 with Covid-19 would survive the infection based on age and other risk factors [9]. Data-Based

129 Analysis, Modelling and Forecasting infection rates and spread/patient prognosis to enable

130 hospitals/health officials to better plan resourcing and response [10].

131

132 Machine learning technologies are used to improve the accuracy of prediction for screening

133 both infectious and non-infectious diseases [11]. The machine learning methods were used in 134 modeling former pandemics (e.g., Ebola, Cholera, swine fever, H1N1 influenza [12], dengue 
135 fever [13], Zika, oyster norovirus [14]). Table 1 represents notable Machine Learnings methods used for outbreak prediction.

Table 1. Notable Machine Learning techniques for outbreak prediction

\begin{tabular}{|c|c|c|c|}
\hline References & $\begin{array}{l}\text { Outbreak } \\
\text { infection }\end{array}$ & $\begin{array}{l}\text { Machine learning } \\
\text { methods }\end{array}$ & Resources used to collect data \\
\hline $\begin{array}{l}\text { Geospatial } \\
\text { Health [15] }\end{array}$ & Dengue fever & Neural Network & $\begin{array}{l}\text { The computerized database for } 2016 \text { has } \\
\text { been obtained from Taiwan Centers for } \\
\text { Disease Control (Taiwan CDC, 2016), } \\
\text { which contains all national dengue fever } \\
\text { records daily from 1998, including age, } \\
\text { gender, township of residence and time of } \\
\text { disease onset. }\end{array}$ \\
\hline $\begin{array}{l}\text { Malaysian } \\
\text { Journal of } \\
\text { Public Health } \\
\text { Medicine [16] }\end{array}$ & Dengue/Aedes & Bayesian Network & $\begin{array}{l}\text { The Aedes outbreak database sourced } \\
\text { from the Ministry of Health at pre- } \\
\text { determined localities around the Klang } \\
\text { Valley, Malaysia. }\end{array}$ \\
\hline $\begin{array}{l}\text { Global Ecology } \\
\text { and } \\
\text { Biogeography } \\
{[12]}\end{array}$ & H1N1 flu & Neural Network & $\begin{array}{l}\text { Data on the prefectural distribution of the } \\
\text { H1N1 influenza pandemic in } 2009 \text { were } \\
\text { provided by the Ministry of the } \\
\text { Environment of Japan. }\end{array}$ \\
\hline $\begin{array}{l}\text { Current Science } \\
{[17]} \\
\text { Infectious } \\
\text { Disease } \\
\text { Modelling [18] }\end{array}$ & Dengue & $\begin{array}{lr}\text { Naïve Bayes } & \\
\text { Classification } & \text { and } \\
\text { regression } & \text { tree } \\
\text { (CART) } & \end{array}$ & $\begin{array}{l}\text { The data has been collected on the monthly } \\
\text { dengue cases from the Government of the } \\
\text { National Capital Territory (NCT) of Delhi } \\
\text { (India). }\end{array}$ \\
\hline $\begin{array}{l}\text { Environment } \\
\text { International } \\
{[19]}\end{array}$ & Oyster norovirus & Neural Network & $\begin{array}{l}\text { The epidemiological data were obtained } \\
\text { from various online data sources. } \\
\text { Specifically, historical norovirus } \\
\text { outbreaks in Louisiana oyster harvest } \\
\text { areas were collected from Louisiana } \\
\text { Morbidity Reports released by Louisiana } \\
\text { Department of Health and Hospitals }\end{array}$ \\
\hline
\end{tabular}


140 Another recent study presents a comparative analysis of machine learning and soft computing

141 models to predict the COVID-19 outbreak as an alternative to Susceptible- Infectious-Removed

142 (SIR) and Susceptible-Exposed-Infectious-Removed (SEIR) models. Among a wide range of

143 machine learning models investigated, two models showed promising results (i.e., multi-

144 layered perceptron, MLP, and adaptive network-based fuzzy inference system, ANFIS) [20].

145 Also, Machine learning and deep learning can replace humans by giving an accurate diagnosis

146 [21]. The perfect diagnosis can save radiologists' time and can be cost-effective than standard

147 tests for COVID-19. X-rays and computed tomography (CT) scans can be used for training the

148 machine learning model. Several initiatives are under-way in this regard. Wang and Wong

149 developed COVID-Net, which is a deep convolutional neural network, which can diagnose

150 COVID-19 from chest radiography images [22]. Moreover, Recent studies [28] show the

151 potential of Artificial Intelligence and Machine Learning tools by suggesting a new model that

152 comes with rapid and valid method SARS-CoV-2 diagnosis using Deep Convolutional Network

$153[23,24]$. In this regard, the remarkable performance suggests the use of the convolutional neural

154 network (Resnet-101) as an adjuvant tool for increase the accuracy of Covid-19 diagnosis.

155 Recent studies used the supervised machine learning techniques for classifying the text into

156 four different categories COVID, SARS, ARDS and Both (COVID, ARDS). Logistic

157 regression and Multinomial Naïve Bayes showed better results than other ML algorithms for

158 detecting COVID-19 using clinical text data set [25].

159

160 In a comparative analysis framework between various supervised machine learning algorithms

161 in diagnosing COVID-19 infections, Pijush Dutta implemented bagging algorithm, k-nearest

162 neighbor, and random forest for classifying the datasets of COVID-19 [26]. Random Forest

163 gave better results with employing accuracy of $85.71 \%$. Haochen Yao and Nan Zhang built a

164 COVID-19 severeness detection model based on supervised machine learning algorithms [27], 
and obtained the best model using the Support Vector Machine algorithm (SVM) with 28

166 features and overall accuracy of $81.48 \%$. Mahbubunnabi Tamal trained a supervised machine

167 learning algorithms to distinguish between COVID-19 and other diseases, where he found that

168 SVM and Ensemble Bagging Model Trees (EBM) when trained on 71 radiomics features can

169 distinguish between COVID-19 and other diseases with an overall sensitivity of $99.6 \%$ and

$17087.8 \%$ and specificity of $85 \%$ and $97 \%$ respectively [28]. Davide Brinati developed two

171 machine learning classification models using hematochemical values from routine blood exams

172 to discriminate between patients who are either positive or negative to the SARS-CoV-2 [29]:

173 their accuracy ranges between $82 \%$ and $86 \%$, and sensitivity between $92 \%$ and $95 \%$.

175 Due to the highly complex nature of the COVID-19 outbreak and variation in its behavior from

176 nation-to-nation, this study suggests applying the machine learning as an effective tool to model

177 the detection and improve the accuracy of prediction for screening coronavirus disease

178 (COVID-19) according to the time of their evolution (in days) in different country. Various

179 supervised machine learning algorithms exist in the literature. The best-known are Linear

180 Discriminant Analysis (LDA) [30], Naïve Bayes Classification (NBC) [31], Decision Tree

181 Classification (DTC) [32, 33], and Support Vector Machine Classification (SVM) [34],

182 Artificial Neural Network (ANN) [35]. Since we collected mixed data, I strongly suggested

183 comparing the classifiers available for mixed data, such as Extended Gamma Associative

184 Classifier (EG) [36], Naive Associative Classifier (NAC) [37]. Irrespective of the method

185 chosen, the use of Cross-validation is a model assessment technique used to evaluate a

186 supervised classification algorithm's performance in making predictions on new COVID-19

187 datasets that it has not been trained on. This paper aims to investigate the generalization ability

188 of the proposed Machine Learning models and the accuracy of the proposed models for 
detecting the degrees of possibility of Coronavirus disease infection from official reports of

190 COIVD-19 data.

\section{Mathematical Methods}

193 The goal of supervised learning is to build a concise model of the distribution of class labels in

194 terms of predictor features. The resulting classifier is then used to assign class labels to the

195 testing samples where the values of the predictor feature are known, but the value of the class

196 labels are unknown. The classifier's evaluation is most often based on prediction accuracy (the

197 percentage of correct prediction divided by the total number of predictions) [38]. There are at

198 least three techniques which are used to calculate a classifier's accuracy. One technique is to

199 split the available data by using part of them for training and the other for estimating 200 performance, for example two-thirds for training and the other third for testing. In the second 201 technique, known as cross-validation, the training set is divided into mutually exclusive and 202 equal sized subsets and for each subset the classifier is trained on the union of all the other subsets. The average of the error rate of each subset is therefore an estimate of the error rate of 204 the classifier. The third technique is the leave one out validation, which is a special case of cross 205 validation. All test subsets consist of a single sample. This type of a validation is more 206 expensive computationally, but useful when the most accurate estimate of classifier's error rate 207 is required.

208 Supervised classification is one of the tasks most frequently carried out by so-called artificial 209 intelligence. Many techniques have been developed based such as Logic-based techniques, 210 Perceptron-based techniques, Bayesian Networks, etc. In this application, we have chosen 211 Decision Trees classifiers as logic learning methods, and Linear Discriminant Analysis, 212 Support Vector Machines and Naïve Bayes Classification as statistical learning algorithms. 213 These techniques are widely used in several research areas, such as biology, medicine fields 
214 and advanced technology [35]. Conversely to artificial neural network, statistical approaches

215 are characterized by having an explicit underlying probability model, which provides a 216 probability that a sample belongs in each class, rather than simply a classification. In addition,

217 the perceptron-based techniques require several parameters which must be studied such that the 218 size of hidden layers and number of neurons. For this, it is difficult to adapt the neural network 219 method as an objective function in the methods of selection of variables such as Genetic 220 Algorithms.

221 Let $\mathbf{X}=\left\{\mathbf{x}_{\mathbf{j}}\right\}_{j=1}^{n}$ be a training set of $n$ samples of observed variables, where each sample is 222 represented by an S-dimensional vector and let $c_{k}$ denoting the class membership of $\mathbf{x}_{\boldsymbol{j}}$, where $c_{k} \in\left\{c_{1}, \ldots, c_{K}\right\}$ with $\mathrm{K}$ denoting the number of classes of training set.

\subsection{Naive Bayesian classifier}

The naïve Bayesian classifier (NBC) is based on Bayes' theorem and predicting independence requirements. A naïve Bayesian model is simple to construct since it does not need costly iterative parameter estimates, resulting in a quick computing time for training [39].

Given $\mathbf{x}_{j}=\left[x_{j 1}, \ldots, x_{j s}, \ldots, x_{j S}\right]^{T} \in \mathbb{R}^{S}$, selection of observed data retrieved from the training data set $\mathrm{X}$ and belonging to the class $c_{k}$. The Naïve Bayes classifier can predict that $\mathbf{x}_{\boldsymbol{j}}$ belongs to the predicted class $\hat{C}$ with the following Maximum a Posteriori Probability (MAP) based on $\mathbf{x}_{j}:$

$$
\operatorname{MAP}\left(\mathbf{x}=\mathbf{x}_{j}\right)=\arg \max _{k \in\{1, \ldots, K\}} P\left(C=c_{k} \mid \mathbf{x}=\mathbf{x}_{\boldsymbol{j}}\right)
$$

234 According to Bayes' theorem, the probability $P\left(C=c_{k} \mid \mathbf{x}=\mathbf{x}_{j}\right)$ that we wish to calculate 235 could be defined in terms of $P\left(C=c_{k}\right), P\left(\mathbf{x}=\mathbf{x}_{\boldsymbol{j}} \mid C=c_{k}\right)$ and $P\left(\mathbf{x}=\mathbf{x}_{\boldsymbol{j}}\right)$ as

$$
P\left(C=c_{k} \mid \mathbf{x}=\mathbf{x}_{j}\right)=\frac{P\left(\mathbf{x}=\mathbf{x}_{j} \mid C=c_{k}\right) P\left(C=c_{k}\right)}{P\left(\mathbf{x}=\mathbf{x}_{j}\right)},
$$


238 value of $C$, and the value of $P\left(\mathbf{x}=\mathbf{x}_{\boldsymbol{j}}\right)$ is a known constant. $P\left(\mathbf{x}=\mathbf{x}_{\boldsymbol{j}} \mid C=c_{k}\right)$ is called the 239 Class-conditional Probability Distribution (CPD). Thus, [40, 41] gives the computation of the 240 Nave Bayes classifier:

$$
\operatorname{MAP}\left(\mathbf{x}=\mathbf{x}_{\boldsymbol{j}}\right)=\arg \max _{\mathrm{k} \in\{1, \ldots, K\}} P\left(\mathbf{x}=\mathbf{x}_{\boldsymbol{j}} \mid \mathrm{C}=c_{k}\right) P\left(C=c_{k}\right) \text {. (eq. 3) }
$$

242 The likelihood of a classification error, often known as the classifier's risk, is defined as [31]:

$$
R^{N B C}\left(\mathbf{x}_{\boldsymbol{j}}\right)=\sum_{\boldsymbol{x}_{j} \in \mathbf{X}} \mathbf{1}_{C\left(\mathbf{x}=\mathbf{x}_{j}\right) \neq \hat{C}\left(\mathbf{x}_{=\mathbf{x}_{j}}\right)} P\left(\boldsymbol{X}=\boldsymbol{x}_{j}\right)=E_{\boldsymbol{X}}\left\{\mathbf{1}_{C\left(\mathbf{x}=\mathbf{x}_{j}\right) \neq \hat{C}\left(\mathbf{x}=\mathbf{x}_{j}\right)}\right\}, \text { (eq. 4) }
$$

244 where $\mathbf{1}_{\mathrm{C}\left(\mathbf{x}=\mathbf{x}_{j}\right) \neq \widehat{\mathrm{C}}\left(\mathbf{x}=\mathbf{x}_{j}\right)}$ is the indicator function and $E_{\boldsymbol{X}}$ is the expectancy over $\mathbf{X}$ and $245 \mathrm{C}\left(\mathbf{x}=\mathbf{x}_{j}\right)$ is the real class which the vector $\mathbf{x}_{j}$ corresponds and $\hat{C}\left(\mathbf{x}=\mathbf{x}_{j}\right)$ is the expected 246 class of $\mathbf{x}_{\boldsymbol{j}}$ provided by the NBC method. The Bayes error is the rate of misclassification (Bayes 247 risk). When addressing classification issues, the Bayes risk is frequently specified as the 248 reference. It is the least error rate when the distribution is given.

\subsection{Linear Discriminant Analysis}

Linear Discriminant Analysis (LDA) is a dimensional reducing and classification approach that has been widely used to the analysis of spectral data. Consider the challenge of allocating a sample of observed variables retrieved from the training set $\mathbf{x}_{\boldsymbol{j}}$ to the class $c_{k}$ in a classification task. The categorization score is calculated as follows:

$$
c f\left(\mathbf{x}_{\boldsymbol{j}}\right)=\left(\mathbf{x}_{\boldsymbol{j}}-\mu_{k}\right)^{T} \Sigma_{\mathrm{k}}^{-1}\left(\mathbf{x}_{\boldsymbol{j}}-\mu_{k}\right)+\ln \left|\Sigma_{\mathrm{k}}\right|-2 \ln \left[P\left(C=c_{k}\right)\right]
$$
and $\mu_{k}$ is the mean vector of class $c_{k}$, and they are calculated by

$$
\widehat{\mu_{k}}=\frac{1}{n_{k}} \sum_{j=1}^{n_{k}} \mathbf{x}_{j}
$$

$$
\widehat{\Sigma_{k}}=\frac{1}{n_{k}} \sum_{j=1}^{n_{k}}\left(\mathbf{x}_{\boldsymbol{j}}-\mu_{k}\right)\left(\mathbf{x}_{\boldsymbol{j}}-\mu_{k}\right)^{T}
$$

$$
P\left(\widehat{C=} c_{k}\right)=\frac{n_{k}}{n}
$$

where $n_{k}$ represents the number of samples in class $c_{k}$, and $\mathrm{n}$ represents the total number of 
samples in the training dataset. The sample $\mathbf{x}_{\boldsymbol{j}}$ is classified as belonging to the class with the

263 lowest classification score [32].

264 When the class covariance matrices are believed to be equal, a pooled covariance matrix is 265 formed.

$$
\Sigma_{\text {pooled }}=\frac{1}{n} \sum_{k=1}^{K} n_{k} \Sigma_{k}
$$

267 and is substituted for the class covariance matrix in eq. (5). Without taking into account constants, the following classification rule for LDA is produced.

$$
c f\left(\mathbf{x}_{j}\right)=\left(\mathbf{x}_{j}-\mu_{k}\right)^{T} \Sigma_{\text {pooled }}^{-1}\left(\mathbf{x}_{j}-\mu_{k}\right)-2 \ln \left[P\left(C=c_{k}\right)\right]
$$

Equation (10) relates to the Mahalanobis distance when the prior probability $n_{i}$ is constant. The

LDA classifier's error rate $R^{L D A}$, which is defined as:

$$
R^{L D A}\left(\mathbf{x}_{\boldsymbol{j}}\right)=\frac{1}{n} \sum_{j=1}^{n}\left|\operatorname{sign}\left(\mathrm{c}_{k}\left(\mathbf{x}_{\boldsymbol{j}}\right)-\hat{c}_{k}\left(\mathbf{x}_{\boldsymbol{j}}\right)\right)\right|
$$

273

with $\boldsymbol{x}_{\boldsymbol{j}}$ belonging to the class $c_{k}$ as determined by the LDA method classifier in the class $\hat{c}_{k}$

\subsection{Decision Tree Classifier}

A decision tree classifier is a non-parametric classifier that does not require any a priori statistical assumptions to be made regarding the distribution of data [43]. The basic structure of the decision tree, however, consists of one root node, a number of internal nodes and finally a set of terminal nodes [44]. A node is a subset of the predictors that is used to determine a split. A non-terminal node or parent node is a node that is further split into two child nodes. Growing a tree consists of selecting the optimal splits to determine a non-terminal node, and the assignment of each terminal node to a class. The data is recursively divided down the decision tree according to the defined classification framework.

Classes are simply assigned to a terminal node by observing which class is mostly commonly observed in that region of the tree. Thus, the challenge is to optimally choose the best variable and split that variable to maximize the purity or similarity among the responses. The impurity 
of a parent node $\tau$, denoted $i(\tau)$, is zero when all observations are in the same class. A split s

287 is determined by selecting the best predictor and split value that optimizes the highest reduction 288 in purity $[45,46]$

$$
\Delta(s, \tau)=i(\tau)-\sum_{b=1}^{B} p\left(\tau_{b} / \tau\right) i\left(\tau_{b}\right)
$$

where $\tau_{b}$ denotes child node $\mathrm{b}, p\left(\tau_{b} / \tau\right)$ is the proportion of observations in $\tau$ that are assigned entropy criterion [33]

$$
i(\tau)=-\sum_{k=1}^{K} p_{k} \log _{2}\left(p_{k}\right)
$$

and the Gini index criterion

$$
i(\tau)=-\sum_{k=1}^{K} p_{k}^{2}
$$

where $p_{k}$ is the proportion of observations in class $c_{k}$ with $k \in\{1, \ldots, \mathrm{K}\}$. Pruning is based upon successive steps of removing lower branches that lead to improved classification rates. by comparing the observed class to the predicted class from the CT for observation $\mathbf{x}_{\boldsymbol{j}}$. In a terminal node $m$, representing a region $R_{m}$ with $n_{m}$ observations, let

$$
\hat{p}_{m k}=\frac{1}{n_{m}} \sum_{j=1}^{n_{m}} \mathbf{1}_{c_{k}}\left(\mathbf{x}_{\boldsymbol{j}}\right)
$$

denote the proportion of class $c_{k}$ observations in terminal node $\mathrm{m}$. We classify the observations in node $m$ to class

$$
\widehat{c_{k}}\left(\mathbf{x}_{j}\right)=\underset{\mathrm{k}}{\operatorname{argmax}} \hat{p_{m k}}
$$

The misclassification error rate is simply the proportion of observations in the node that are not members of the majority class in that node.

$$
R^{D T C}\left(\mathbf{x}_{j}\right)=\frac{1}{n} \sum_{j=1}^{n}\left(1-\max _{k}\left(\hat{p_{m k}}\left(\mathbf{x}_{j}\right)\right)\right.
$$


311 Support vector machines (SVMs) are a type of learning algorithms that are used for 312 classification and regression. SVM classifiers, like Decision Trees, are non-parametric [47]. 313 The one-against-all technique [48] is now the most fundamental technique for implementing 314 SVM multi-class classification. K binary SVM models are built in this simplest application of 315 the SVM to a K-class issue. Class $\mathrm{c} k$ is isolated from the other classes in the kth class SVM 316 problem. To create a final multi-class classifier, all k binary SVM classifiers are concatenated.

317 The term "remaining" refers to the fact that all data points from classes other than $c_{k}$ are merged 318 to form a single class $c_{l}$. Using the usual SVM technique [42], the ideal hyperplane that 319 separates data points from the class $c_{i}$ and the combined class $c_{l}$ is obtained. The best separating hyperplane distinguishing the classes $c_{i}$ and $c_{k}$ is denoted as:

$$
g_{k}\left(\mathbf{x}_{j}\right)=\mathbf{w}_{\boldsymbol{k}} \cdot \varphi\left(\mathbf{x}_{j}\right)+\mathbf{b}_{\boldsymbol{k}} \quad \mathbf{k} \in\{1, \ldots, \mathrm{K}\}
$$

322 where $\mathbf{w}_{\boldsymbol{k}} \in \mathbb{R}^{S}$ is the weight vector, $\mathrm{b}$ is the bias, and the transfer function $\varphi$ maps the training 323 data into an appropriate feature space $\mathbb{R}^{S}$ to allow for complex nonlinear surfaces. The 324 following minimization is used to estimate the parameters of the decision function $g_{k}\left(\mathbf{x}_{\boldsymbol{j}}\right)$ :

$$
\min J\left(\mathbf{w}_{\boldsymbol{k}}, \xi\right)=\frac{1}{2}\left\|\mathbf{w}_{\boldsymbol{k}}\right\|^{2}+C \sum_{j=1}^{n} \xi_{j}
$$

326 subject to

$$
y_{j}\left(\mathbf{w}_{\mathbf{k}}^{\mathrm{T}} \varphi\left(\mathbf{x}_{j}\right)+\mathbf{b}_{\boldsymbol{k}}\right) \geq \mathbf{1}-\xi_{j} \quad \xi_{j} \geq \mathbf{0} ; j=1 \ldots \mathrm{n}, \quad(\text { eq. } 20)
$$

328 To loosen the separability restrictions in with scalar $y_{j} \in\{-1,+1\}$ signifying its class label, $\mathrm{C} \in \mathbb{R}^{+}$is a regularization constant, and $\xi_{j}$ is a slack variable (eq. 19). The $f_{k}\left(\mathbf{x}_{j}\right)$ decision rule, which allocates the vector $\mathbf{x}_{j}$ to the class $c_{k}$ provided by:

$$
f_{k}\left(\mathbf{x}_{\mathbf{j}}\right)=\operatorname{sign}\left(g_{i}\left(\mathbf{x}_{\mathbf{j}}\right)\right)
$$

332 The fundamental challenge with this strategy is that the classifier outputs $f_{k}\left(\mathbf{x}_{j}\right)$ are binary numbers. The standard approach to this problem is to disregard the sign-operator in eq. 21 . We 
say $\mathbf{x}_{j}$ is in the class with the biggest value of the decision function and is provided by after

335 finding all the optimum hyperplanes given by $g_{k}\left(\mathbf{x}_{\mathbf{j}}\right)$ for $\mathbf{k} \in\{1, \ldots, \mathrm{K}\}$.

$$
\widehat{c_{k}}\left(\mathbf{x}_{j}\right)=\underset{k}{\operatorname{argmax}} g_{k}\left(\mathbf{x}_{j}\right)
$$

337 The index of the biggest component of the different classifiers $g_{i}\left(\mathbf{x}_{\boldsymbol{j}}\right)$ for $\mathbf{k} \in\{1, \ldots, \mathrm{K}\}$ is 338 allocated to the data point $\mathbf{x}_{\boldsymbol{j}}$ in this manner. The SVM classifier's error rate $R^{S V M}$, which is 339 defined as:

$$
R^{S V M}\left(\mathbf{x}_{\boldsymbol{j}}\right)=\frac{1}{n} \sum_{j=1}^{n} \mathbf{1}_{c_{k} \neq \hat{c}_{k}}\left(\boldsymbol{x}_{\boldsymbol{j}}\right)
$$

341 with $\boldsymbol{x}_{\boldsymbol{j}}$ belonging to the class $c_{k}$ as assessed by the method classifier in the class $\hat{c}_{k}$ and $\mathbf{1}_{c_{k} \neq c_{k}}\left(\boldsymbol{x}_{\boldsymbol{j}}\right)$ being the indicator function defined as:

$$
\mathbf{1}_{c_{k} \neq \hat{c}_{k}}\left(\boldsymbol{x}_{\boldsymbol{j}}\right)=\left\{\begin{array}{l}
1 \text { if class } \hat{c}_{k} \text { of } \boldsymbol{x}_{\boldsymbol{j}} \neq \text { class } c_{k} \\
0 \text { if class } \hat{c}_{k} \text { of } \boldsymbol{x}_{\boldsymbol{j}}=\operatorname{class} c_{k}
\end{array}\right.
$$

\subsection{Artificial Neural Network}

Artificial Neural network (ANN) method is an algorithm in machine learning and artificial intelligence, which is inspired by the human nervous system to analyze and model complex, nonlinear systems, and parallel computations [49]. Haykin introduces an artificial neural network as a massively parallel learning machine, made up of simple processing units called neurons [50].

A neuron has asset of inputs $x_{1}, x_{2}, \ldots, x_{R}$ and each connection from the inputs to the processing 353 element is affected by different connection strengths known as synaptic weights. A neuron is 354 showed in mathematical and schematic terms by the following equations $(25,26)$ and Figure 1.

$$
\Sigma=w_{1} x_{1}+w_{2} x_{2}+w_{3} x_{3}+\cdots+w_{n} x_{n}+b
$$

$$
y=f(\Sigma)
$$


357 Where $x_{1}, x_{2}, \ldots, x_{n}$ are the inputs and $w_{1}, w_{2}, \ldots, w_{n}$ are the synaptic weights of the neuron

358 L. $b$ is the bias, $f$ is the activation function, and $y$ is the output of the neuron.

360

361

362

363

364

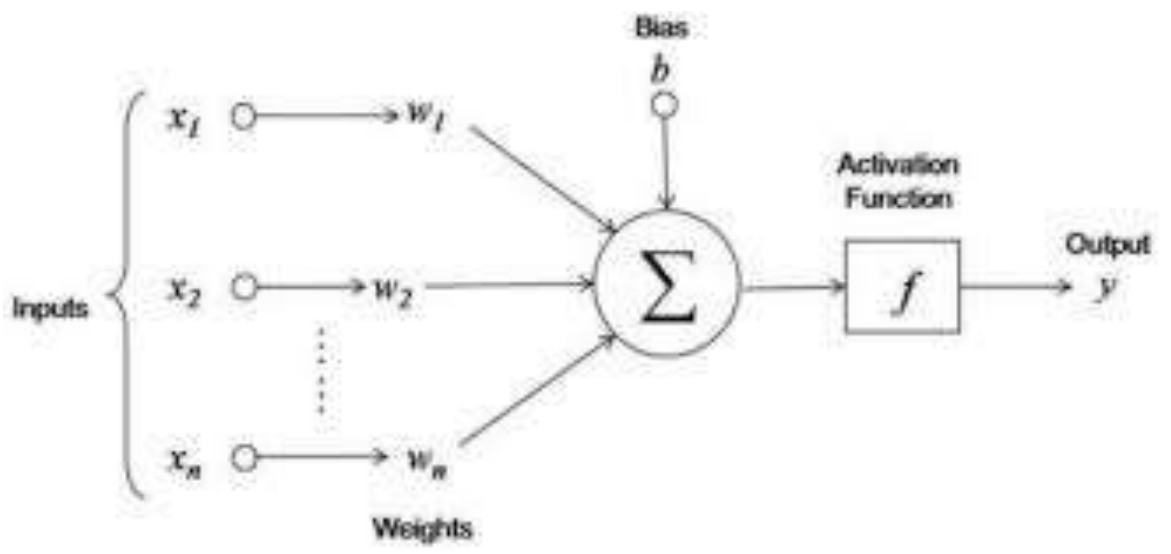

Figure 1. Structure of a neuron

The output of the neuron is defined by applying an activation function to the linear combination of inputs and weights. There are many different activation functions as shown in Figure 2.

Sigmoid

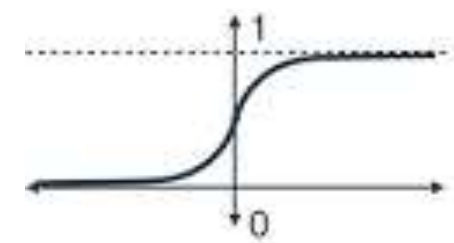

Hyperbolic tangent (tanh)

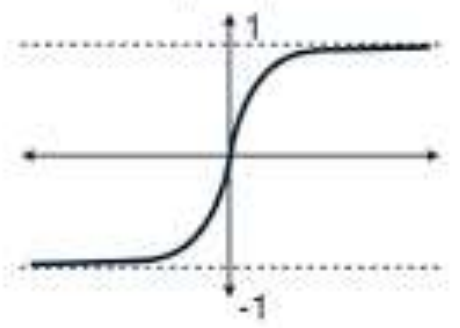

Rectified linear unit (ReLU)

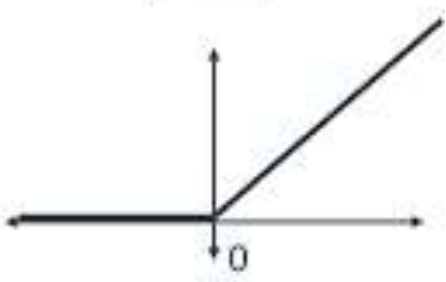

Figure 2. Different activation functions

The procedure in which neurons are arranged in a neural network is known as the network topology or architecture. There are many different types of neural network such as MLP, RBF and PNN. One of the famous ANN architecture which is most widely used in remote sensing application is multilayer perceptron, or MLP that has been used in this study. An MLP is a feed- 
374 forward neural network with one or more layers of neurons between the input and output layer

375 called hidden layers [51].

376

377

394 where

\subsection{Extended Gamma Associative Classifier}

The Gamma Associative Classifier (EG) is a supervised learning used as prediction model for classification. This algorithm has able to handle mixed data, or absence of information in the data. The extended gamma association classifier (EG) includes two stages: training and classification. Let $\mathrm{X}$ and $\mathrm{Y}$ be the training and testing datasets, respectively, where each instance $x_{j} \in \mathrm{X}, y_{j} \in \mathrm{P}$ is described by a set of attributes or factors. [36]

The training stage of the classifier starts with the storage of the training set and incorporates the calculations of various parameters such that the pause and stop parameters. Within the classification stage, EG employments an iterative handle, based on the calculation of the average similarity with each class of the samples to be classified [52]. To analyze the similitude between test sample and training sample, the extended generalized similitude $\gamma_{\text {ext }}$ is utilized. After getting the similitudes, the average of the generalized similarity of said test pattern for each class $c_{k}$ is calculated. The number of samples belonging to the class $\mathrm{k}$ in the training data is given by $\mathrm{n}$, and $x_{i j}$ represents the value of the $\mathrm{j}$-th attribute of the $\mathrm{i}$-th sample of the class $c_{k}$, and $w_{j}$ represents the weight of the jth feature with $w_{j} \in\left[\begin{array}{ll}0 & 1\end{array}\right]$.

$$
c_{k}=\frac{\sum_{i=1}^{n} \sum_{j=1}^{m} w_{j} \gamma_{e x t}\left(x_{j}, y_{j}, \theta\right)}{n}
$$

$$
\gamma_{\text {ext }}\left(x_{j}, y_{j}, \theta\right)=\left\{\begin{array}{lr}
\gamma_{n u m}\left(x_{j}, y_{j}, \theta\right) & \text { if } \mathrm{jth} \text { feature is numeric } \\
\gamma_{\text {cat }}\left(x_{j}, y_{j}\right) & \text { if } \mathrm{jth} \text { feature is categoric } \\
\gamma_{\text {miss }}\left(x_{j}, y_{j}\right) & \text { if } \mathrm{x}_{\mathrm{j}} \text { or } \mathrm{y}_{\mathrm{j}} \text { are missing }
\end{array}\right.
$$

$$
\gamma_{\text {num }}\left(x_{j}, y_{j}, \theta\right)=\left\{\begin{array}{rr}
1 & \text { if }\left|x_{j}-y_{j}\right| \leq \theta \\
0 & \text { otherwise }
\end{array}\right.
$$




$$
\begin{aligned}
& \gamma_{c a t}\left(x_{j}, y_{j}\right)= \begin{cases}1 & \text { if } \mathrm{x}_{\mathrm{j}}=\mathrm{y}_{\mathrm{j}} \\
0 & \text { otherwise }\end{cases} \\
& \gamma_{\text {miss }}\left(x_{j}, y_{j}\right)= \begin{cases}1 & \text { if } \mathrm{x}_{\mathrm{j}}=\mathrm{y}_{\mathrm{j}}=" ? " \\
0 & \text { otherwise }\end{cases}
\end{aligned}
$$

398 In case a single greatest is found among all the values of $c_{k}$, the process ends. If not, the values 399 of the stop and pause parameters, as well as the value of the $\theta$ parameter, are taken under 400 consideration in an iterative process [53].

401

\subsection{Naïve Associative Classifier}

403 The Naïve Associative Classifier (NAC) a novel supervised learning model, directly handles 404 mixed and incomplete data [37]. In its training stage, the NAC stores the training set and 405 calculates, for each numerical attribute, the standard deviation. However, the NAC classifier 406 needs to store the entire training set of data. It also uses similarity comparisons with respect to the entire training set to classify a new sample [54].

408 In the classification stage, the Mixed and Incomplete Data Similarity Operator (MIDSO) and 409 the total similarity operator are used to analyze the similarity between the test samples and the training samples $s^{t}(x, y)$.

$$
S^{t}(x, y)=\sum_{i=1}^{m} w_{i} * \operatorname{MIDSO}\left(x, y, A_{i}\right)
$$

$$
\operatorname{MIDSO}\left(x, y, A_{i}\right)=\left\{\begin{array}{l}
s_{n}\left(x, y, A_{i}\right) \text { if } A_{i} \text { is numeric } \\
s_{c}\left(x, y, A_{i}\right) \text { if } A_{i} \text { is categoric }
\end{array}\right.
$$

$$
s_{c}\left(x, y, A_{i}\right)=\left\{\begin{array}{r}
0 \text { if }\left(\left(\mathrm{x}_{\mathrm{j}} \neq \mathrm{y}_{\mathrm{j}}\right) \vee\left(\mathrm{x}_{\mathrm{j}}=^{\prime} ?^{\prime}\right) \vee\left(\mathrm{y}_{\mathrm{j}}={ }^{\prime} ?^{\prime}\right)\right) \\
1 \quad \text { otherwise }
\end{array}\right.
$$

$$
s_{n}\left(x, y, A_{i}\right)=\left\{\begin{array}{rr}
0 & \text { if }\left(\left(\left|\mathrm{x}_{\mathrm{j}}-\mathrm{y}_{\mathrm{j}}\right|>\sigma_{i}\right) \vee\left(\mathrm{x}_{\mathrm{j}}=^{\prime} ?^{\prime}\right) \vee\left(\mathrm{y}_{\mathrm{j}}={ }^{\prime} ?^{\prime}\right)\right) \\
1 & \text { otherwise }
\end{array}\right.
$$


417 With $w_{i}$ represents the weight of the ith attribute. After having obtained the similarities, we 418 calculated the average of the generalized similarity of the test instance for each class kl, noted $419 s_{l}()$.

$$
s_{l}(.)=\frac{1}{n} \sum_{y \in k_{l}} s^{t}(., y)
$$

421 If a single maximum is found among all the values of $s_{l}($.$) , the process ends. If not, any of the$ classes with maximum similarity is assigned [55].

\section{Application and results}

In this section, we first present data collection that contains the several indicators that directly or indirectly affects the spread of the coronavirus pandemic. The discrimination analysis of samples of infected patients using the proposed approach of supervised classification algorithms is presented and discussed regarding the size of training and validation dataset. All data treatments were performed using the MATLAB ${ }^{\circledR}$ R2019b environment, and scripts are available upon request.

\subsection{Data set collection}

433 The essential pavement procedure to examine the test hypothesis and to evaluate the predicted 434 outcome is in collecting and processing the data. Thus, to predict the behavior of coronavirus spread, four countries have been chosen. These countries have adopted different methodologies to deal with the coronavirus pandemic and achieved different results related to the methodology used: China, Lebanon, Italy, and Iran. The date range for the data for each of the four countries 438 is as the following:

439 China: 9 January 2020 - 28 March 2020 (80 days)

440 Lebanon: 21 February 2020 - 31 March 2020 (40 days)

441 Italy: 31 January 2020 - 31 March 2020 (61 days) 
444 After studying the situation of the virus in these countries, we noticed that there are several

445 indicators that affect directly or indirectly the spread of the coronavirus. These indicators are 446 presented as follows :

447 - The governance reactions: the different responses and reactions from governments of 448 the four countries during the outbreak. These indices are used to explore whether the government response affects the rate of infection and identify correlates of intense responses.

- The medical resources: This factor refers to the health system policies such as the COVID-19 testing regime or emergency investments into healthcare (ICU beds...) and the health services quality in these four countries. The sensitivity effects of this factor on the results are proposed to be investigated in this study.

- The quarantine commitment of the people in the country with the government Guidelines. This factor must have theoretically a straight depending on relation with the variation of the spread intensity of the virus between the countries.

- The special events: this factor takes into consideration the existence of simultaneous events that affect the spread of the coronavirus: other disasters, economic problems, war, political problems or disturbance, official holidays, etc.

- The economical level and governmental aids: This factor refer to the economic policies, such as income support to citizens or the provision of foreign aid. Depending on the direct relationship between this factor and the quarantine commitment of the people in the country, we have proposed it to be present in this study.

- Previous experience history of the four governments that determines the existence of experience in such kind of critical disaster management or not. 
- The use of technology devoted to control the virus spread in these countries that help in the health and hospitalization services, the lockdown control, and the restrictions of the infected zones.

- The population age average/ The population density which is considered as the number of the people in one $\mathrm{km}^{2}$ / in the four countries which causes a meaningful variation in the situation of the infected records.

These direct and indirect factors are considered as parameters used by our model to predict the future behavior of the spread of COVID-19. The data is combined into a series of novel indices that aggregate various measures of each factor. Parameters are measured depending on a specific criterion presented in the following table 2 :

Table 2. The direct and indirect factors that affecting the spreading level of the coronavirus in different countries.

\begin{tabular}{|c|c|c|}
\hline Effective factors & Indications & Values \\
\hline \multirow{5}{*}{ Governance reactions (Lockdown) } & No lockdown measures at all (value $=0$ ). & \multirow{5}{*}{$0,0.25,0.5,0.75$, and 1} \\
\hline & $\begin{array}{l}\text { Disabling major facilities: Stop flights at } \\
\text { airports, public transport, ports, tourist places, } \\
\text { universities and school closures and restrictions } \\
\text { in movement in the public places (value }=0.25 \text { ) }\end{array}$ & \\
\hline & Partially lockdown measures (value $=0.5$ ) & \\
\hline & Fully lockdown measures (value=0.75) & \\
\hline & Providing virus checks abundantly (value $=1$ ) & \\
\hline \multirow{4}{*}{ Medical resources } & $\begin{array}{c}0=\text { no } \text { medical resources, } 0.25=\text { low, } 0.5= \\
\text { medium, } 0.75=\text { good, } 1=\text { high }\end{array}$ & \multirow{4}{*}{$0,0.25,0.5,0.75$, and 1} \\
\hline & - Number of nurses for 1000 people & \\
\hline & - Number of doctors for 1000 people & \\
\hline & - $\quad$ Number of icu beds for 1000 & \\
\hline Previous experience history & yes / no & $1 / 0$ \\
\hline
\end{tabular}




\begin{tabular}{|c|c|c|}
\hline The used technology & $\begin{array}{c}0=\text { no technology, } 0.25=\text { low, } 0.5=\text { medium, } \\
0.75=\text { good, } 1=\text { high }\end{array}$ & $0,0.25,0.5,0.75$, and 1 \\
\hline Special events & yes / no & $1 / 0$ \\
\hline $\begin{array}{c}\text { Economical resources and } \\
\text { governmental aids }\end{array}$ & $\begin{array}{c}0=\text { no economic resources, } 0.25=\text { low, } 0.5= \\
\text { medium, } 0.75=\text { good, } 1=\text { high }\end{array}$ & $0,0.25,0.5,0.75$, and 1 \\
\hline Population density / km2 & $*$ & Number \\
\hline Family number & $*$ Number \\
\hline Quarantine commitment \\
(Procedure)
\end{tabular}

481 Thus, we have collected real data for the four countries, from different official sources with all 482 parameters and daily records (recovered, death, confirmed, PCR tests).

484 Many factors have been known to be associated with the initial levels of the Coronavirus 485 outbreak at the country level including geographical factors [56], demographical parameters 486 [57], healthcare services [58], and economic status [59].

487 As for the technique of data collection, we relied on studies conducted in these four countries 488 to obtain them. In Iran, the demographical data were extracted from the Statistical Centre of 489 Iran [60], healthcare services and economic levels were extracted from [61]. The number of 490 daily confirmed cases was obtained from the Iranian Ministry of Health and Medical Education 491 website.

492 In China, these factors have been selected using data from the Figshare site, nationwide survey 493 in China and World Health Organization (WHO) [62]. In Italy, the collected data were extracted 494 from the most recently updated databases on the website of the Italian Institute of Statistics 
(ISTAT) [63], and the ISTAT's Health for All database [64, 65]. In Lebanon, the data has been

496 collected from the Ministry of Health [66], Interior and Finance to obtain these factors and the 497 number of daily confirmed cases.

498 In another study, we conducted a Path analysis which is a statistical technique that uses both 499 bivariate and multiple linear regression techniques to test the causal relations among the 500 variables specialized in the model [67]. We evaluated the parameter estimates of the previously 501 stated regressors on covid-19 daily cases to assess the most influential factors that contribute to 502 the prediction of day-by-day covid-19 infections. The results showed that quarantine commitment $(\beta=-0.823)$ and full lockdown measures $(\beta=-0.775)$, has the largest direct effect 504 on covid-19 daily infections. The results also show that more experience $(\beta=-0.35)$, density 505 in society $(\beta=-0.288)$, medical resources $(\beta=0.136)$, and economy resources $(\beta=0.142)$ have 506 indirect effects on covid-19 daily infections.

507 These direct and indirect factors allow to measure the score of each sample which represents 508 the times in days in four countries: China, Iran, Italy, and Lebanon. The daily records are 509 basically the cumulative records for a dependent day-by-day scale. In other words, the record 510 of the next day is the sum of the records of the current day and the new records obtained in the 511 same day. The samples were classified into 3 categories which are the degrees of possibility of 512 Coronavirus disease infection according to their evolution (in days): Low (if the standardized 513 cumulative cases are between 0 and 0.09), medium (if the standardized cumulative cases are 514 between 0.09 and 0.3 ) and High (if the standardized cumulative cases are above 0.3 ). The data 515 collected is an imbalanced data meaning that a certain degree of possibility of infection is 516 dominant over other degrees, where in our case the low-risk degree dominates the other two 517 degrees. This is due to the data collected at the beginning of the covid-19 pandemic where most 518 countries are still at low risk of infection and the virus is not fully spread. Regarding the distribution of the degree of possibility of getting infected by covid-19 among the countries 
studied, China (80 days) and Lebanon (40 days) remained at low risk for the whole durations

521 while Iran maintained a low risk of infection in the first 20 days, medium risk of infection for 522 the next 15 days and high risk of infection for the last 7 days of the 42 total days studied in this 523 country. As for Italy, it remained at a low risk for the first 36 days, medium risk for the next 7 524 days, and high risk for the rest 18 days of the 61 total days studied in Italy.

525 In addition, the data collected do not include missing values. The application of supervised 526 learning methods may result in better accuracy, unless a missing value is expected to have a very high variance. All Covid-19 data were preprocessed with a Standard Normal Variate (SNV) preprocessing method [68].

\subsection{Analysis of discrimination of samples of infected patients by the Covid-19}

To deepen the obtained results, we analyzed the confusion matrices and the ROC curves. A confusion matrix is used to describe the performance of a classification model on a set of test data for which the true values are known. It is a summary of prediction results on a classification

534 problem and allows the visualization of the performance of an algorithm. Hence, the elements 535 in the diagonal are the elements correctly classified, while the elements out of the diagonal are 536 misclassified. It can easily be seen that the overall accuracy can be computed by dividing the sum of the diagonal elements (number of correctly classified samples) by the total sum of the elements of the matrix (total number of samples in the dataset). 
A Confusion Matrix

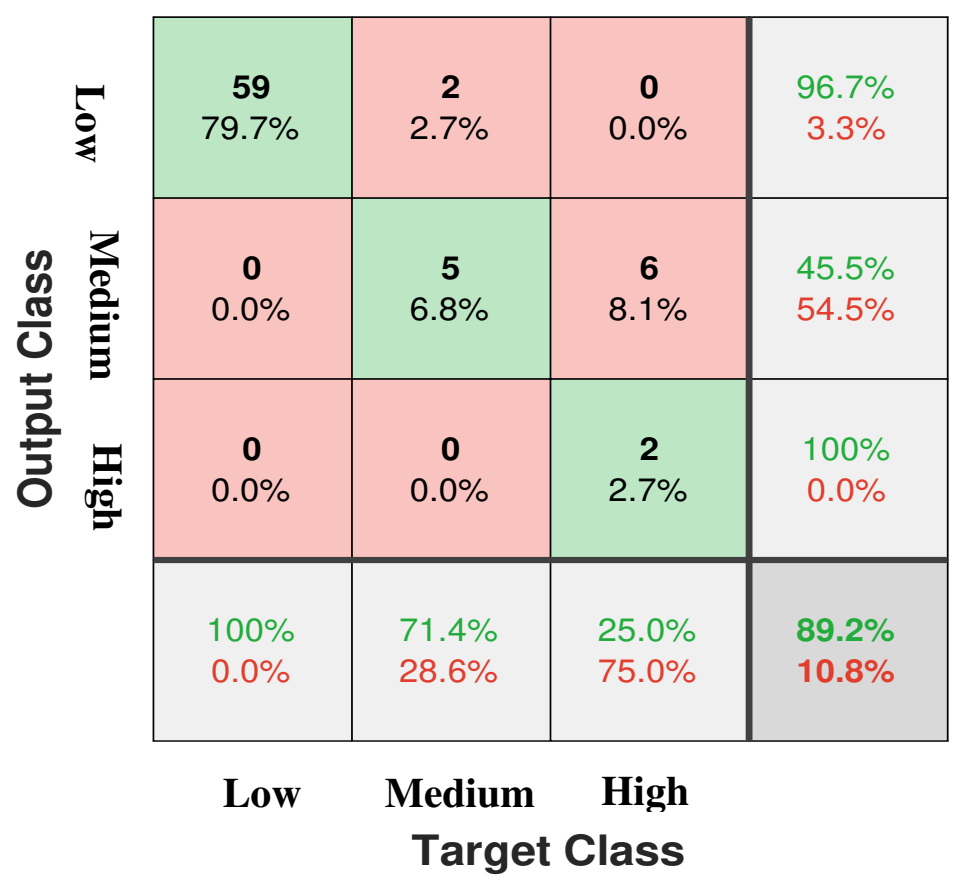

C Confusion Matrix

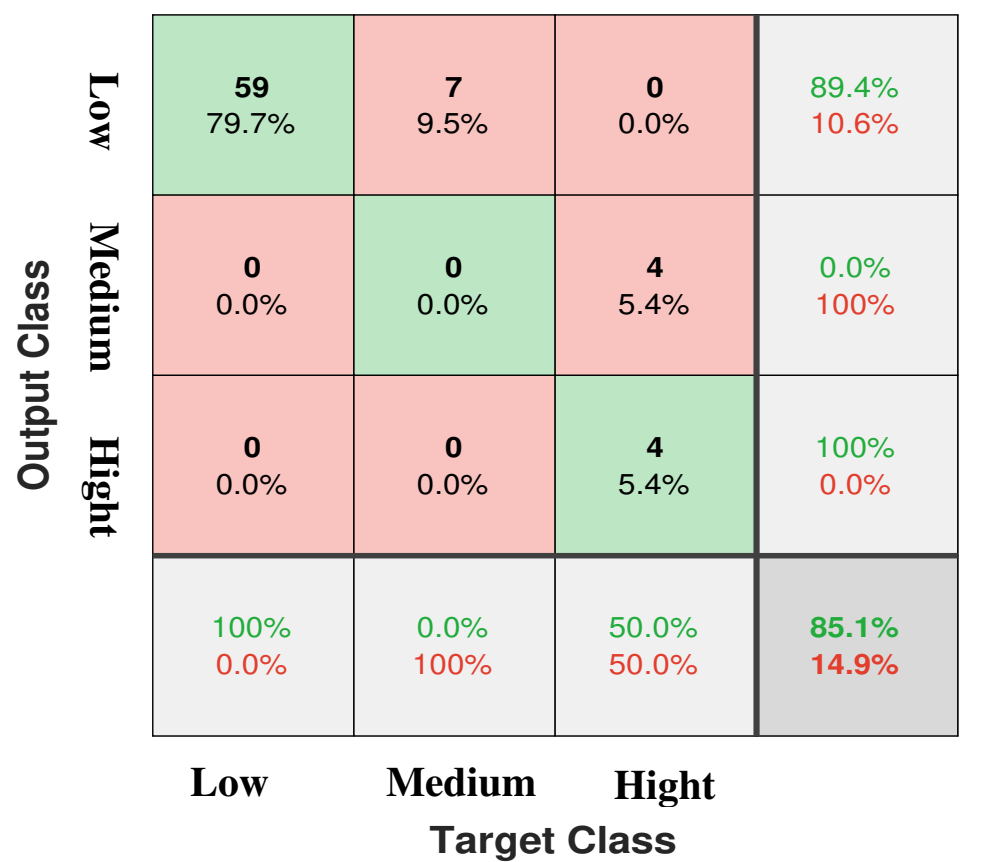

540

\section{B Confusion Matrix}

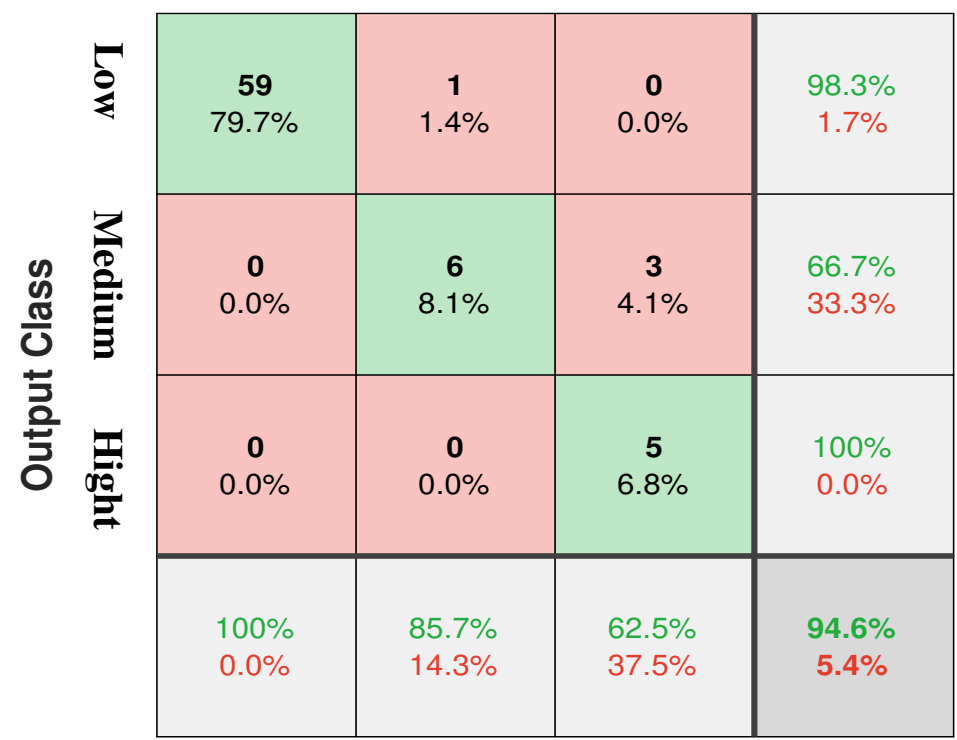

Low Medium High

Target Class

D Confusion Matrix

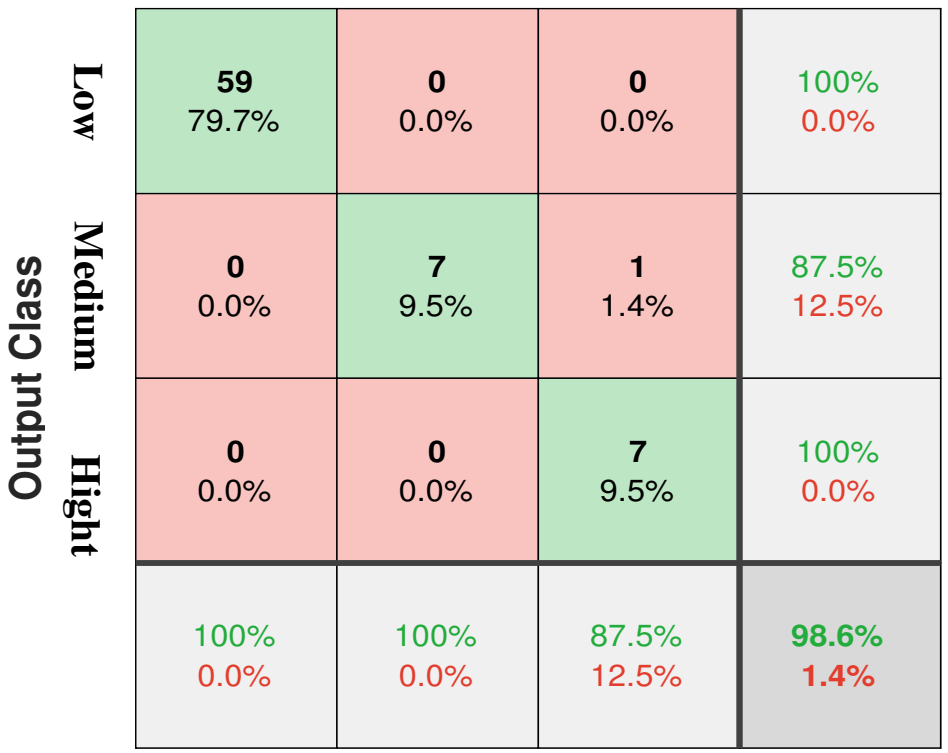

Low Medium Hight

Target Class 
E Confusion Matrix

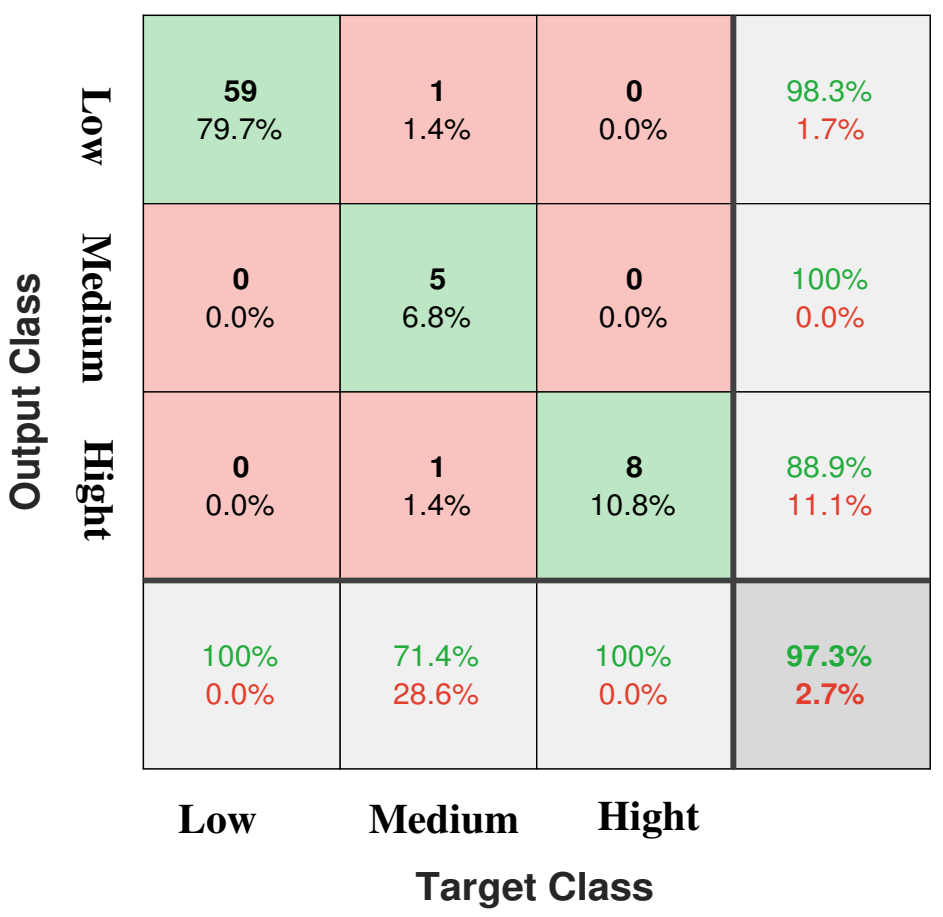

544
F Confusion Matrix

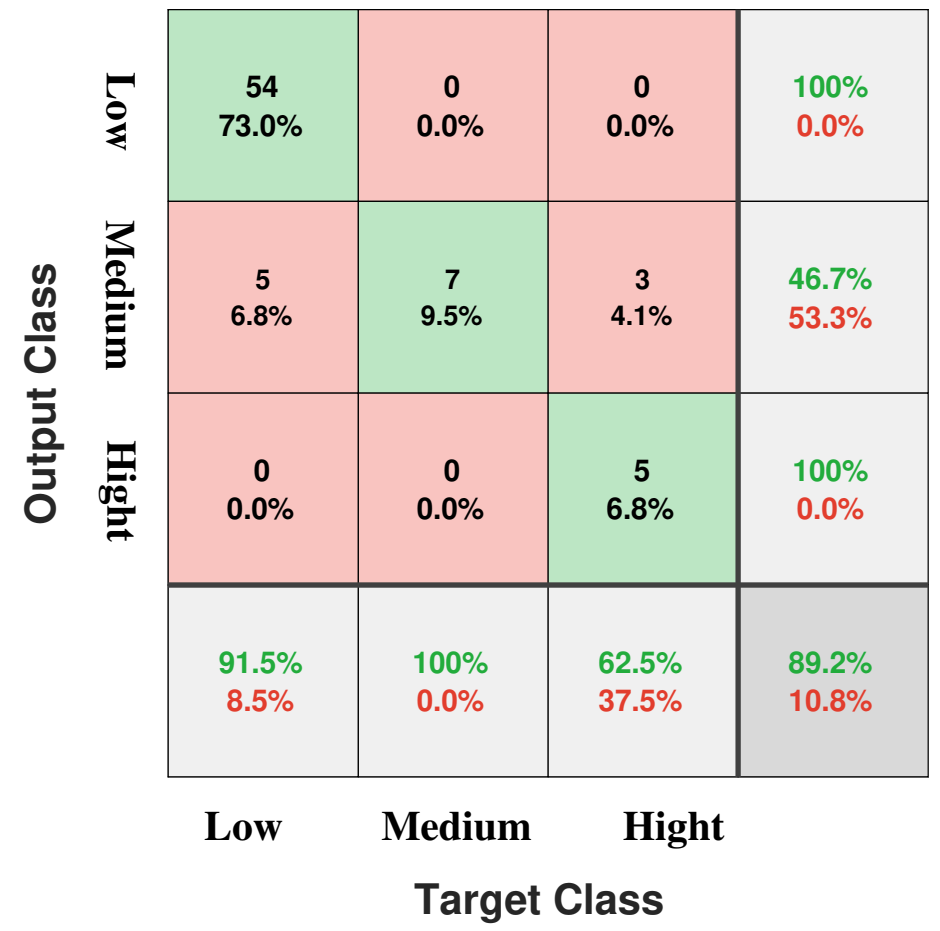

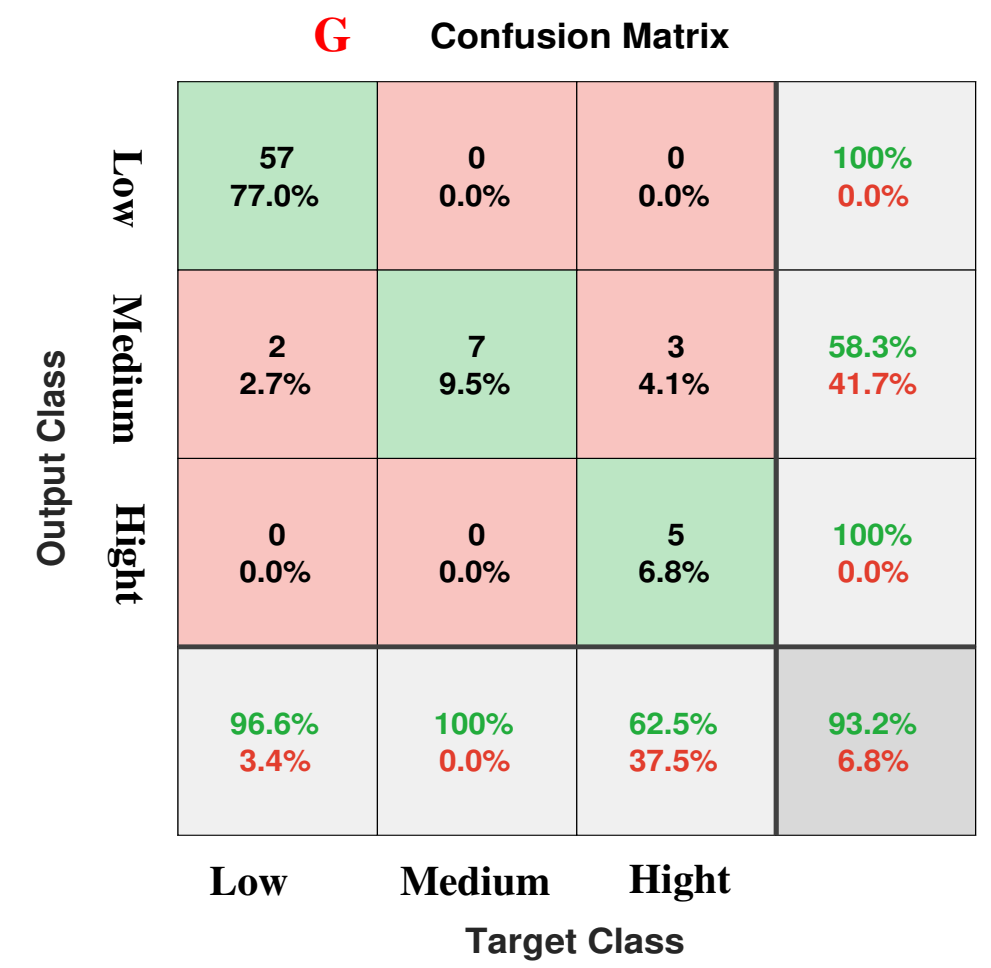

546 Table 2. Results of average confusion matrix (of 100 runs) for the training (2/3 of samples) and

547 testing (1/3 of samples) dataset using the supervised algorithms (A) LDA (B) NBC (C) SVM 
(D) DTC (E) ANN (F) EG (G) NAC methods for the detection of infected patients by the Covid-

54919 according to the time of evolution (in days) of coronavirus disease in different country

Cross-validation is a model assessment technique used to evaluate a supervised classification algorithm's performance in making predictions on new datasets that it has not been trained on. This is done by partitioning a dataset and using a subset to train the algorithm and the remaining data for testing. Several methods of cross validation exist such as k-fold, Holdout, Leave-out and Re-substitution. We use k-fold method that partitions samples of infected patients by the

Covid-19 into $\mathrm{k}=2$ until 10 randomly chosen subsets of roughly equal size. With this approach there is not a possibility of high bias if we have limited data, because we would not miss some information about the data which we have not used for training. the discussion of choosing the value of $\mathrm{K}$ in $\mathrm{K}$-fold cross validation, is shown in section 5.3. but here we will display the results of $K=3$-fold. One subset is used to validate the model $(1 / 3$ of data $=74$ samples $)$ trained using the remaining subsets $(2 / 3$ of data $=149$ samples $)$. This process is repeated $\mathrm{T}=100$ times to get the best stable model and to ensure that most of our samples of infected patients by the Covid-19 have been used for validation.

The Linear Discriminant Analysis (LDA), Naïve Bayes Classifier (NBC), Support Vector Machine (SVM), Decision Tree Classifier (DTC), Artificial Neural Network (ANN), Extended

566 Gamma Associative Classifier (EG) and Naive Associative Classifier (NAC) proposed 567 supervised algorithms has been applied on the coronavirus data. Tables $2(A)(B)(C)(D)(E)$ presents the performance of the LDA, NBC, SVM, DTC and ANN classifications as the mean over the $\mathrm{T}=100$ samplings of the confusion matrix information for the samples of infected 570 patients by the Covid-19 according to the time of evolution (in days) of coronavirus disease in 571 different country. Each table show the confusion matrix for the true label's targets and predicted 572 labels outputs. The rows correspond to the predicted class (Output Class) extract by 
574 (Target Class). We have three degrees of possibility according to their evolution of coronavirus 575 that represents the classes of our COIVID-19 data: Low, Medium and Hight. The diagonal cells correspond to spectra that are correctly classified. The off-diagonal cells correspond to incorrectly classified of degree of infected samples (time of evolution) by the Covid-19.

The results of classification accuracy for Detection True Classifier (DTC) and Artificial Neural 579 Networks (ANN) algorithms registered $98.6 \%$ and $97.4 \%$ of validation datasets describing the 580 degree of possibility of Coronavirus disease infection. However, the classification accuracy is 581 worse for the Naïve Bayes Classifier (NBC) algorithm with $94.6 \%$ of validation datasets 582 correctly classified, the Support Vector Machine (SVM) algorithm with $85.1 \%$ of validation 583 datasets correctly classified and Linear Discriminant Analysis (LDA) algorithm with $89.8 \%$ of 584 validation datasets correctly classified. These values, presented in Table 3, confirm that the 585 DTC and ANN algorithm allows a better discriminate within the degrees of possibility of 586 Coronavirus disease infection according to days of their evolution than state of art methods of 587 supervised classifications such as NBC, SVM, EG, NAC and LDA algorithms.

A ROC (Receiver Operating Characteristic) curve summarizes the performance of a classifier 590 over all possible thresholds. It is generated by plotting the True Positive Rate (y-axis) against 591 the False Positive Rate (x-axis) by varying the threshold for assigning observations to a given 592 class. A ROC curve is a performance measurement for classification problem at various 593 thresholds settings. The plot of True Positive Rate (TPR; sensitivity) versus False Positive Rate 594 (FPR; 1-specificity) across varying cut-offs generates a curve in the unit square called a ROC 595 curve. ROC curves are used to compare different supervised classifiers. In our case we have 596 used the multi-class ROC curves, which is a kind of multi-objective optimization to compare 597 the supervised classification algorithm; LDA, NBC, SVM, ANN, DTC, EG and NAC. As in 598 several multi-class problem, the idea is generally to carry out pairwise comparison: one class 
vs. all other classes, one class vs. another class [69]. If the curve has high values, it leads to the

600 greater of area under the curve obtained, and the less error the classifier makes. ROC curve 601 corresponding to progressively greater discriminant capacity are located progressively closer

602 to the upper left-hand corner in "ROC space". To numerically assess the discrimination of the 603 classes, the Area Under the Curve (AUC) was used to measure of the ability of a classifier to 604 distinguish between classes and is used as a summary of the ROC curve. Higher the AUC, 605 better the model is at distinguishing between classes of degrees of possibility of Coronavirus 606 disease.

607 The figure below represents the comparisons between ROC curves by LDA, NBC, SVM, ANN 608 and DTC for each class of degrees of possibility of Coronavirus disease infection according to 609 days of their evolution for validation datasets (1/3 samples). The ROCs of each model are 610 plotted using the average of 100 runs. We can see that the classification threshold in ANN and 611 DTC methods (figures 3D and 3E) are very close to 1 and very far from diagonal for all the 612 classes of degrees of possibility of Coronavirus disease compared to other methods. But the 613 classification threshold is relatively reduced for the LDA and SVM methods (figures 3A and 614 3B). The area under the curves of each class of degrees of possibility of Coronavirus disease 615 for the ANN and DTC methods are higher than that for NBC, SVM, LDA, EG and NAC 616 methods. This also confirms that the measures of sensitivity and specificity of ROC curves for 617 ANN and DTC algorithms are generally stronger than the other ROC curves for NBC, SVM, 618 LDA EG and NAC algorithms for each class of degrees of possibility of Coronavirus disease 619 in all figures $3(\mathrm{~A}-\mathrm{G})$, which indicates better in-sample accuracy than the other classifier 620 methods. The AUC values, presented in Figures 3 (A-G), confirm that the ANN and DTC 621 supervised methods allows a better classifier of degrees of possibility of Coronavirus disease. 

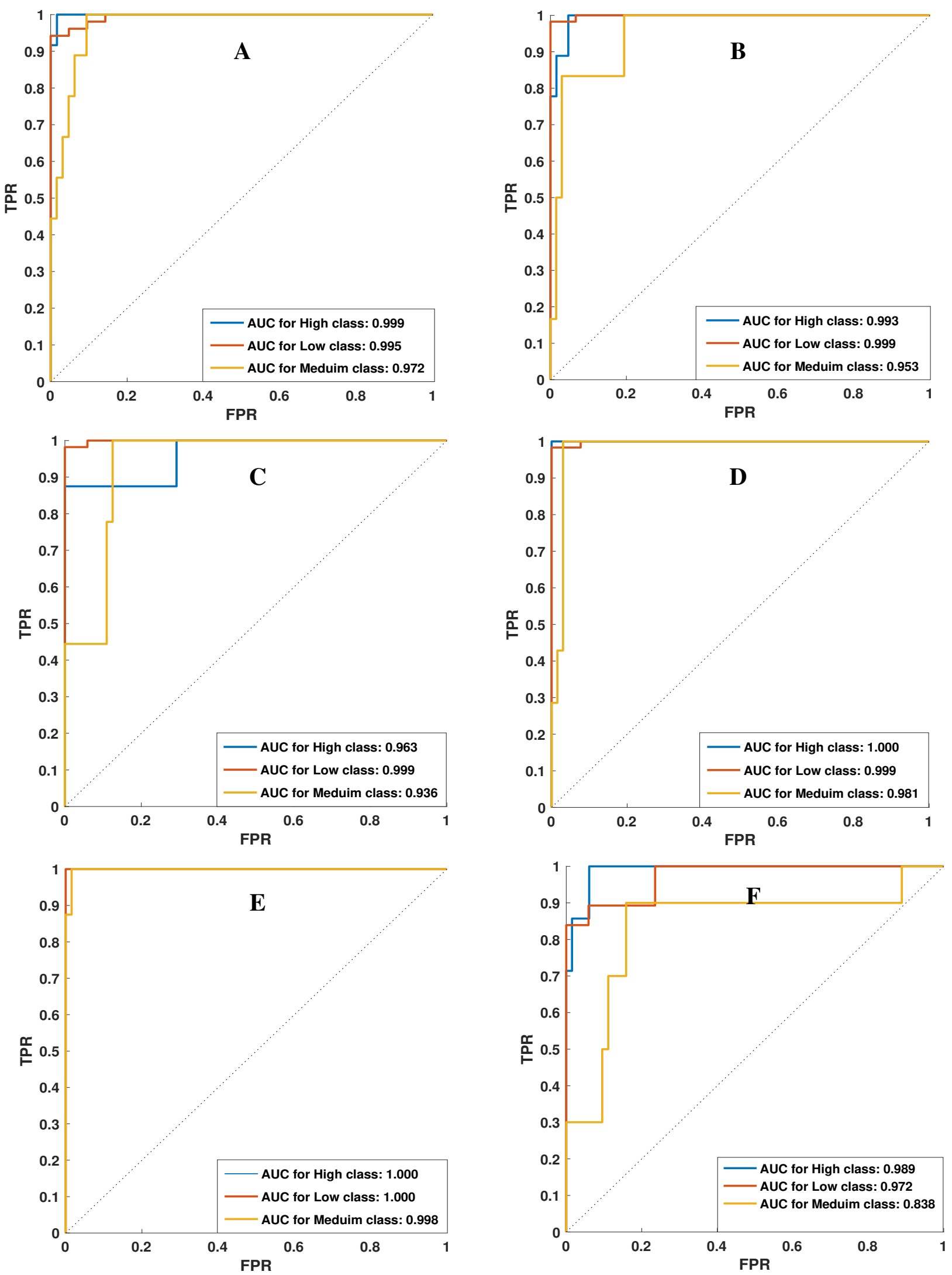


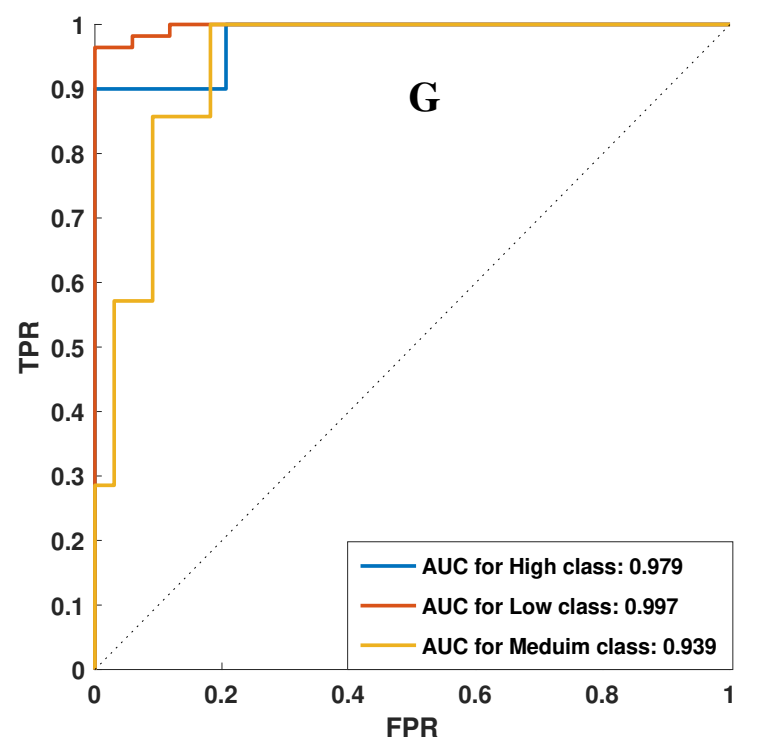

625

626 Figure 3. The average of 100 iterations of Receiver operating characteristic (ROC) curve of A) LDA, (B) NBC, (C) SVM, (D) ANN and (E) DTC (F) EG (G) NAC supervised classifications algorithms for classify the degrees of possibility of Coronavirus disease infection according to days of their evolution using the validation datasets.

630

632 As we already mentioned, cross validation is the most important part of machine learning

633 models, and the results might depend on it. K-fold cross-validation is extremely useful with

634 the appropriate $\mathrm{K}$ which is the number of folds chosen.

635 Unfortunately, there is no theoretically perfect procedure of determining the appropriate value 636 of $\mathrm{K}$ in $\mathrm{K}$-fold cross validation. Many researchers used $\mathrm{K}=10$, for example, see Bengio and 637 Grandvalet (2004), Vehtari and Lampinen (2004). Davison and Hinkley (1997) recommend K $638=\min \left(\mathrm{n}^{1 / 2}, 10\right)$ in practice. Clark (2003) compares different values of $\mathrm{K}$ for his data and 639 suggested that the choice of $\mathrm{K}=4$ will probably be good in general, though not necessarily 640 optimal. Duan et al. (2003) used $\mathrm{K}=5$ for the number of folds. Anderson et al (2006) 641 suggested, leaving $20 \%$ of the n-samples at a time for final model validation. For this, we have 
642 tested the method of supervised learning classification LDA, NBC, SVM, ANN and DTC on

643 different partitions of testing and training dataset depending on different values of $\mathrm{K}$ between

6442 and 10. To examine the influence of the cross-validation method, we have computed the ratio

645 of classification (\%) obtained for each method of the supervised learning classification LDA,

646 NBC, SVM, ANN and DTC on COVID-19 data. The values in Table 3, indicate that the

647 detection tree classifier (DTC) algorithm gives the best results of classification for different

648 sizes of testing and training datasets for the samples of infected patients by Covid-19 according

649 to the time of evolution (in days) of coronavirus disease in different countries. This sampling

650 analysis is done to assess the importance of K-fold cross-validation method in determining the

651 best classifier among the classifiers studied.

652

653 Table 3. The Means and Standard Deviation of classification performance (expressed as \%) for 100 runs of LDA, NBC, SVM, ANN, DTC, EG and NAC supervised methods on different sizes

validation.

\begin{tabular}{|c|c|c|c|c|c|c|c|c|}
\hline & K-fold & LDA & $\mathrm{NBC}$ & SVM & ANN & DTC & EG & NAC \\
\hline $\begin{array}{l}1 / 2 \text { training \& } 1 / 2 \\
\text { testing }\end{array}$ & 2 & $87.4 \pm 2.7$ & $91.4 \pm 2.2$ & $82.3 \pm 4.5$ & $91.2 \pm 2.8$ & $96.9 \pm 1.7$ & $\begin{array}{l}83.1 \pm \\
5.2\end{array}$ & $\begin{array}{l}91.8 \pm \\
3.3\end{array}$ \\
\hline $\begin{array}{l}2 / 3 \text { training \& } 1 / 3 \\
\text { testing }\end{array}$ & 3 & $89.5 \pm 3.4$ & $94.5 \pm 2.5$ & $85.6 \pm 5.2$ & $97.3 \pm 1.8$ & $98.6 \pm 0.7$ & $\begin{array}{l}89.2 \pm \\
4.2\end{array}$ & $\begin{array}{l}93.2 \pm \\
2.6\end{array}$ \\
\hline $\begin{array}{l}3 / 4 \text { training \& } 1 / 4 \\
\text { testing }\end{array}$ & 4 & $89.5 \pm 3.2$ & $94.5 \pm 1.9$ & $87.1 \pm 4.4$ & $98.1 \pm 0.8$ & $98.9 \pm 0.5$ & $\begin{array}{l}90.4 \pm \\
3.8\end{array}$ & $\begin{array}{l}93.3 \pm \\
2.6\end{array}$ \\
\hline $\begin{array}{l}4 / 5 \text { training \& } 1 / 5 \\
\text { testing }\end{array}$ & 5 & $90.2 \pm 3.4$ & $95.4 \pm 2.6$ & $87.4 \pm 3.8$ & $\begin{array}{l}99.1 \pm \\
0.5\end{array}$ & $99.2 \pm 0.3$ & $\begin{array}{l}91.2 \pm \\
3.8\end{array}$ & $\begin{array}{l}93.5 \pm \\
2.4\end{array}$ \\
\hline $\begin{array}{l}9 / 10 \text { training \& } 1 / 10 \\
\text { testing }\end{array}$ & 10 & $91.1 \pm 2.8$ & $95.8 \pm 2.2$ & $88.9 \pm 3.8$ & $\begin{array}{ll}99.5 \quad \pm \\
0.2\end{array}$ & $99.5 \pm 0.3$ & $\begin{array}{l}91.4 \pm \\
3.2\end{array}$ & $\begin{array}{l}94.5 \pm \\
2.2\end{array}$ \\
\hline
\end{tabular}

657 
659 To quantify the results of the AUROC of each model performed 100 runs, we calculated in The

660 Table 4 the mean and standard deviation of the AUROC for three classes of degrees of 661 possibility of infection with Coronavirus disease infection and the proposed supervised learning 662 methods.

663

664 Table 4. The mean and SD of the AUROC for three classes of degrees of possibility of 665 Coronavirus disease infection.

\begin{tabular}{|l|l|l|l|}
\hline & Low & Medium & High \\
\hline LDA & $0.995 \pm 0.004$ & $0.972 \pm 0.024$ & $0.999 \pm 0.001$ \\
\hline NBC & $0.999 \pm 0.001$ & $0.953 \pm 0.042$ & $0.993 \pm 0.006$ \\
\hline SVM & $0.999 \pm 0.001$ & $0.936 \pm 0.064$ & $0.963 \pm 0.032$ \\
\hline DTC & $0.999 \pm 0.001$ & $0.981 \pm 0.018$ & $1.000 \pm 0.000$ \\
\hline ANN & $1.000 \pm 0.000$ & $0.998 \pm 0.001$ & $1.000 \pm 0.000$ \\
\hline EG & $0.972 \pm 0.027$ & $0.838 \pm 0.158$ & $0.989 \pm 0.001$ \\
\hline NAC & $0.979 \pm 0.026$ & $0.939 \pm 0.058$ & $0.979 \pm 0.028$ \\
\hline
\end{tabular}

666

667 In application of machine learning algorithms for classification tasks, we need to apply 668 approximate statistical test for determining whether one learning algorithm out-performs 669 another on a particular learning task. For this we propose to apply McNemar's test that is based 670 on a X2 test for goodness of fit. So, we may reject the null hypothesis in favor of the hypothesis 671 that the two algorithms have different performance if P-value is greater than 0.05 . We found 672 that the application of McNemar test between seven used supervised algorithms, gives the 673 existence of performance difference between DTC and ANN algorithms and other algorithms, 674 and there is no performance difference between DTC and ANN algorithms. 
COVID-19 has shocked the world due to the speed of its spread and its non-availability of vaccine or drug. Various researchers are working for conquering this deadly virus. We used 224 reports recorded on specified dates in four countries which are labelled in three classes of 680 degrees of infections for each day: Low, medium and Hight possibility of Coronavirus disease 681 infection. Various direct and indirect factors like the economical level and governmental aids, 682 medical resources, previous experience, Governance reactions and the used technology are being extracted from these COVID-19 samples according to the time of evolution (in days) of coronavirus disease in different countries.

685

The machine learning algorithms are used for classifying daily samples in four countries into three different classes degrees of possibility of Coronavirus disease infection. After performing classification, it was revealed that decision trees classifier (DTC) and artificial neural networks (ANN) gives excellent results by having $98.6 \%$ and $97.3 \%$ accuracy well classified. Other machine learning algorithm that showed better results were Naive Bayesian classifier (NBC) by having $94.5 \%$ accuracy well classified. The efficiency of models can be improved by increasing the amount of data.

693

694 Further studies should be performed to investigate some issues, for example, a study that takes 695 into account a priori information such as the degrees of possibility of Coronavirus disease into 696 the objective function through Partial Least Square (PLS) models. Furthermore, it would be 697 very interesting to apply of new technology in the search for the degrees of possibility of 698 Coronavirus disease infection such as the deep learning technique which allows to extract the 699 spectral features to classify the state of COVID-19. 


\section{Acknowledgements}

703 This study was carried out by Faculty of Engineering-Islamic University of Lebanon in

704 partnership with Lebanese University, Statistics and Informatics Department - Faculty of

705 Science in Beirut.

706 Funding

707 This project has been funded with the support of the National Council for Scientific Research

708 in Lebanon CNRS-L.

709 Ethics approval and consent to participate

710 The study does not involve human participants. Not applicable

711 Consent for publication

712 Not applicable

\section{Availability of data and material}

714 All data, models, and code generated or used during the study appear in the submitted article

715 and are provided upon request by contacting Abbas Rammal via email:

716 rammal_abbass@hotmail.com

\section{Competing interests}

718 The authors declare that there are no competing interests.

\section{Authors' contributions}

720 AR: Conceptualization, Methodology, Software, Data curation, Visualization, Validation,

721 Investigation, Original and revised draft preparation, Writing-Reviewing, and Editing. EY:

722 Data curation, Investigation, Original and revised draft preparation, Writing-Reviewing, and

723 Editing. WF and RK: Conceptualization, Methodology, Data Collection, and Writing. All

724 authors read and approved the final manuscript. 
726 [1] Wu, F., Zhao, S., Yu, B., Chen, Y., Wang, W., Song, Z.G., Hu, Y., Tao, Z.W., Tian, J.H., 727 Pei, Y.Y., Yuan, M.L., Zhang, Y.L., Dai, F.H., Liu, Y., Wang, Q.M., Zheng, J.J., Xu, L., 728 Holmes, E.C., Zhang, Y.Z. 2020. A new coronavirus associated with human respiratory disease 729 in china. Nature. 44(59), 265-269.

730 [2] Ardabili, S.F., Mosavi, A., Ghamisi, P., Ferdinand, F., Varkonyi-Koczy, A.R., Reuter, U., 731 Rabczuk, T., Atkinson, P.M. 2020. COVID-19 Outbreak Prediction with Machine Learning 732 http://dx.doi.org/10.2139/ssrn.3580188

733 [3] Ivanov, D. 2020. Predicting the impacts of epidemic outbreaks on global supply chains: A 734 simulation-based analysis on the coronavirus outbreak (COVID-19/SARS-CoV-2) case. 735 Transp. Res. Part E Logist. 136, https://doi:10.1016/j.tre.2020.101922

736 [4] Vaishya, R., Javaid, M., Khan, I.H., Haleem, A. 2020. Artificial Intelligence (AI) 737 applications for COVID-19 pandemic. Diabetes Metab Syndr. 14(4), 337-339.

738 [5] Ai, T., Yang, Z., Hou, H., Zhan, C., Chen, C., Lv, W., Tao, Q., Sun, Z., Xia, L. 2020 739 Correlation of chest CT and RT-PCR testing in coronavirus disease 2019 (COVID-19) in China: 740 a report of 1014 cases. Radiology. 296(2), 32-40.

741 [6] Pham, Q., Nguyen, D.C., Huynh-The, T., Hwang, W., Pathirana, P.N. 2020. Artificial 742 Intelligence (AI) and Big Data for Coronavirus (COVID-19) Pandemic: A Survey on the State743 of-the-Arts. IEEE Access. 8, 130820-130839.

744 [7] Luengo-Oroz, M., Pham, K.H., Bullock, J., Kirkpatrick, R., Luccioni, A., Rubel, S., 745 Wachholz, C., Chakchouk, M., Biggs, P., Nguyen, T., Purnat, T., Mariano, B. 2020. Artificial 746 intelligence cooperation to support the global response to COVID-19. Nature Machine 747 Intelligence. 2, 295-297.

748 [8] Robson, B. 2020. COVID-19 Coronavirus spike protein analysis for synthetic vaccines, a 749 peptidomimetic antagonist, and therapeutic drugs, and analysis of a proposed achilles' heel 750 conserved region to minimize probability of escape mutations and drug resistance. Comput. 
Biol. Med. 121, 103749.

752 [9] Wynants, L., Van Calster, B., Collins, G.S., Riley, R.D., Heinze, G., Schuit, E., Bonten, 753 M.M.J., Damen, J.A.A., Debray, T.P.A., De Vos, M., Dhiman, P., Haller, M.C., Harhay, M.O., 754 Henckaerts, L., Kreuzberger, N., Lohman, A., Luijken, K., Ma, J., Andaur, C.L., Reitsma, J.B., 755 Sergeant, J.C., Shi, C., Skoetz, N., Smits, L.J.M., Snell, K.I.E., Sperrin, M., Spijker, R., 756 Steyerberg, E.W., Takada, T., van Kuijk, S.M.J., van Royen, F.S., Wallisch, C., Hooft, L., 757 Moons, K.G.M., van Smeden, M. 2020. Prediction models for diagnosis and prognosis of covid75819 infection: systematic review and critical appraisal. BMJ. 369.

759 [10] Anastassopoulou, C., Russo, L., Tsakris, A., Siettos, C. 2020 Data-based analysis, 760 modelling and forecasting of the COVID-19 outbreak. PLoS ONE. 15(3).

761 [11] Agrebi, S., Larbi, A. 2020. Use of artificial intelligence in infectious diseases. Artificial 762 Intelligence in Precision Health. 415-438.

763 [12] Koike, F., Morimoto, N. 2018. Supervised forecasting of the range expansion of novel 764 non-indigenous organisms: Alien pest organisms and the $2009 \mathrm{H} 1 \mathrm{~N} 1 \mathrm{flu}$ pandemic. Global 765 Ecol. Biogeogr. 27, 991-1000.

766 [13] Agarwal, N., Koti, S.R., Saran, S., Kumar, S. 2018. Data mining techniques for predicting 767 dengue outbreak in geospatial domain using weather parameters for New Delhi, India. Curr. 768 Sci. 114, 2281-2291.

769 [14] Raja, D.B., Mallol, R., Ting, C.Y., Kamaludin, F., Ahmad, R., Ismail, S., Jayaraj, V.J., 770 Sundram, B.M. 2019. Artificial intelligence model as predictor for dengue outbreaks. Malays. 771 J. Public Health Med. 19, 103-108.

772 [15] Anno, S., Hara, T., Kai, H., Lee, M.A., Chang, Y., Oyoshi, K., Mizukami, Y., Tadono, T. 773 2019. Spatiotemporal dengue fever hotspots associated with climatic factors in taiwan including 774 outbreak predictions based on machine-learning. Geospatial Health. 14, 183-194.

775 [16] Raja, D.R., Mallol, R., Ting, C. Y., Kamaludin, F., Rohani Ahmad, Suzilah Ismail, Vivek 
PREDICTOR FOR DENGUE OUTBREAKS. Malaysian Journal of Public Health Medicine,

778

779

780

781

782

783

784

785

786

787

788

789

790

791

792

793

794

795

796

797

798

799

800 19(2), 103-108.

[17] Agarwal N, Koti SR, Saran S, Kumar AS. Data mining techniques for predicting dengue outbreak in geospatial domain using weather parameters for New Delhi, India. Current Science journal. 2018; 114: 2281-2291.

[18] Titus Muurlink, O., Stephenson, P., Islam, M.Z., Taylor-Robinson, A.W. 2018. Long-term predictors of dengue outbreaks in Bangladesh: A data mining approach. Infectious Disease Modelling. 3, 322-330.

[19] Chenar, S.S., Deng, Z. 2018. Development of artificial intelligence approach to forecasting oyster norovirus outbreaks along Gulf of Mexico coast. Environment International. 111, 212223.

[20] Yang, Z., Zeng, Z., Wang, K., Wong, S.S., Liang, W., Zanin, M., Liu, P., Cao, X., Gao, Z., Mai, Z. 2020. Modified SEIR and AI prediction of the epidemics trend of COVID-19 in China under public health interventions. Journal of Thoracic Disease.

[21] Ozturk, T., Talo, M., Yildirim, E.A., Baloglu, U.B., Yildirim, O., Rajendra Acharya, U. 2020. Automated detection of COVID-19 cases using deep neural networks with X-ray images. Computer Biol Med. 121, 103792.

[22] Wang, L., Wong, A. 2020. COVID-Net: a tailored deep convolutional neural network design for detection of COVID-19 Cases from chest radiography images.

[23] Hemdan, E.E.D., Shouman, M.A., Karar, M.E. 2020. COVIDX-Net: A Framework of Deep Learning Classifiers to Diagnose COVID-19 in X-Ray Images. https://arxiv.org/abs/2003.11055

[24] Wang, S., Kang, B., Ma, J., Zeng, X., Xiao, M., Guo, J., Cai, M., Yang, J., Li, Y., Meng, X., Xu, B. 2020. A deep learning algorithm using CT images to screen for Corona Virus Disease 
801

802

803

804

805

806

807

808

809

810

811

812

813

814

815

816

817

818

819

820

821

822

823

824

825

(COVID-19).

[25] Khanday, A.D., Rabani, S.T., Khan, Q.R. 2020. Machine learning based approaches for detecting COVID-19 using clinical text data. International Journal of Information Technology. $12,731-739$.

[26] Dutta, P., Paul, S., Kumar, A. 2021. Comparative analysis of various supervised machine learning techniques for diagnosis of COVID-19. Electronic Devices, Circuits, and Systems for Biomedical Applications, 521-540. https://doi.org/10.1016/B978-0-323-85172-5.00020-4

[27] Yao, H., Zhang, N., Zhang, R., Duan, M., Xie, T., Pan, J., Peng, E., Huang, J., Zhang, Y., Xu, X., Xu, H., Zhou, F., Wang, G. 2020. Severity Detection for the Coronavirus Disease 2019 (COVID-19) Patients Using a Machine Learning Model Based on the Blood and Urine Tests. Front. Cell Dev. Biol. 8, 683. doi: 10.3389/fcell.2020.00683

[28] Tamal, M., Alshammari, M., Alabdullah, M., Hourani, R., Abu Alola, H., Hegazi, T. 2020. An Integrated Framework with Machine Learning and Radiomics for Accurate and Rapid Early Diagnosis of COVID-19 from Chest X-ray. Expert Systems with Applications, https://doi.org/10.1101/2020.10.01.20205146

[29] Brinati, D., Campagner, A., Ferrari, D., Locatelli, M., Banfi, G., Cabitza, F. 2020. Detection of COVID-19 Infection from Routine Blood Exams with Machine Learning: a Feasibility Study. Journal of Medical Systems, https://doi.org/10.1101/2020.04.22.20075143 [30] Huerta, E.B., Duval, B., Hao, J. K., 2010. A hybrid LDA and genetic algorithm for gene selection and classification of microarray data. Neurocomputing. 73 (13-15), 2375-2383.

[31] Rammal, A., Perrin, E., Vrabie, V., Assaf, R., Fenniri, H. 2017. Selection of discriminant mid-infrared wavenumbers by combining a naïve Bayesian classifier and a genetic algorithm: Application to the evaluation of lignocellulosic biomass biodegradation. Mathematical Biosciences. 289, 153-161.

[32] Punia, M., Joshi, P.K., Porwal, M.C. 2010. Decision tree classification of land use land 
826

827

828

829

830

831

832

833

834

835

836

837

838

839

840

841

842

843

844

845

846

847

848

849

850

cover for Delhi, India using IRS-P6 AWiFS data. Expert Systems with Applications. 38 (5), $5577-5583$.

[33] Safavian, S.R., Landgrebe, D. 1991. A survey of decision tree classifier methodology. IEEE Transactions on Systems, Man, and Cybernetics. 21(3), 660-674.

[34] Liu, M., Wang, M., Wang, D. 2013. Comparison of random forest, support vector machine and back propagation neural network for electronic tongue data classification: Application to the recognition of orange beverage and Chinese vinegar. Sensors and Actuators B: Chemical. $177,970-980$

[35] Kotsiantis, S.B. 2007. Supervised Machine Learning: A Review of Classification Informatica. 31, 249-268.

[36] Villuendas-Rey, Y., Yáñez-Márquez, C., Antón-Vargas, J., López-Yáñez, I. 2019. An Extension of the Gamma Associative Classifier for Dealing with Hybrid Data. Access IEEE, 7, 64198-64205.

[37] Villuendas-Rey, Y., Rey-Benguría, C., Ferreira-Santiago, Á., Camacho-Nieto, O., YáñezMárquez, C. 2017. The naïve associative classifier (NAC): A novel, simple, transparent, and accurate classification model evaluated on financial data. Neurocomputing. 265, 105-115

[38] Kotsiantis, S.B., 2007. Supervised Machine Learning: A Review of Classification Informatica. 31, 249-268.

[39] Rish I. 2001. An Empirical Study of the naive Bayes Classifier, Artificial Intelligence Journal IJCAI. 3: 41-46.

[40] Fukunaga, K. 1990. Introduction to Statistical Pattern Recognition. Academic Press, second edition.

[41] Garber, F.D., Djouadi, A. 1988. Bounds on the Bayes classification error based on pairwise risk functions. IEEE Transactions on Pattern Analysis and Machine Intelligence. 10, 281-288. 
[42] Park, C.H., Lee, M. 2008. On applying linear discriminant analysis for multi-labeled 852 problems. Pattern Recognition Letters. 29 (7), 878-887.

[43] Sharma, R., Ghosh, A., Joshi, P.K. 2013. Decision tree approach for classification of remotely sensed satellite data using open-source support. Journal of Earth System Science, 122, $1237-1247$.

[44] Bressan, G.M., De Azevedo, B.C., Ap, E., Lizzi, S. 2017. A Decision Tree Approach for the Musical Genres Classification. Applied Mathematics \& Information Sciences. 11 (6), 1703 1713.

[45] Rokach, L., Maimon, O. 2008. Data mining with decision trees: Theory and applications, World Scientific. 69, doi.org/10.1142/6604

[46] Tsang, S., Kao, B., Yip, K.Y., Ho, W.S., Lee, S.D. 2009. Decision trees for uncertain data. IEEE 25th International Conference on Data Engineering, 441-444.

[47] Luts, J., Ojeda, F., Plas, R., Van De Moor, B., De Huffel, S. 2010. A tutorial on support vector machine-based methods for classification problems in chemometrics, Analytica Chimica Acta. 665, 129-145.

[48] Cortes, C., Vapnik, V. 1995. Support-vector network. Machine Learning. 20, 273-297, doi.org/10.1007/BF00994018

[49] Jain, A.K., Mao, J., Mohuiddin, K. 1996. Artificial Neural Networks: A Tutorial. IEEE Computer. 29(3), 31-44.

[50] Simon, H. 1999. Neural Networks and Learning Machines. Third Edition. McMaster University Hamilton, Ontario, Canada. Rev. ed of: Neural networks. 2nd ed.

[51] Sildir, H., Aydin, E., Kavzoglu, T. 2020. Design of Feedforward Neural Networks in the Classification of Hyperspectral Imagery Using Superstructural Optimization. Remote Sensing. 12, 956, doi:10.3390/rs12060956

[52] ItzamA, L.Y., Amadeo, J.A.C., Oscar, C.N., Cornelio Y.M. 2011. Pollutants Time-Series 
876 Prediction using the Gamma Classifier, International Journal of Computational Intelligence 877 Systems. 4(4), 680-711, DOI: 10.1080/18756891.2011.9727822

878 [53] Rangel-Diaz-de-la-Vega, A., Villuendas-Rey, Y., Yanez-Marquez, C., Camacho-Nieto, 879 O., Lopez-Yanez, I. 2020. Impact of Imbalanced Datasets Preprocessing in the Performance of 880 Associative Classifiers. Applied Sciences. 10(8), 2779; doi:10.3390/app10082779

881 [54] Rangel-Diaz De La Vega, A., Villuendas-Rey, Y., Yanez-Marquez, C., Camacho-Nieto, 882 O. 2020. The Naïve Associative Classifier With Epsilon Disambiguation. IEEE Access. 8, $883 \quad 51862-51870$.

884 [55] Villuendas-Rey, Y., Hernandez-Castano, J., Camacho-Nieto, O., Yanez-Marquez, C., 885 Lopez-Yanez, I. 2019. NACOD: A Naïve Associative Classifier for Online Data. IEEE Access, $8867,117761-117767$.

887 [56] Sun, Z., Zhang, H., Yang, Y., Wan, H., Wang, Y. 2020. Impacts of Geographic Factors 888 and Population Density on the COVID-19 Spreading under the Lockdown Policies of China. 889 Science of the Total Environment. 746(666).

890 [57] Dowd, J. B., Andriano, L., Brazel, D., Rotondi, V., Block, P., Ding, X., Liu, Y., Mills, M. 891 2020. Demographic Science Aids in Understanding the Spread and Fatality Rates of COVID892 19. Proceedings of the National Academy of Sciences of the United States of America 117(18), $893 \quad 9696-98$

894 [58] Emanuel, E.J., Persad, G., Upshur, R., Thome, B., Parker, M., Glickman, A., Zhang, C., 895 Boyle, C., Smith, M., Phillips, J.P. 2020. Fair Allocation of Scarce Medical Resources in the 896 Time of Covid-19. N Engl J Med. 382(21), 2049-2055. doi: 10.1056/NEJMsb2005114.

897 [59] Lai, C.C., Wang, C.Y., Wang, Y.H., Hsueh, S.C., Ko, W.C., Hsueh, P.R. 2020. Global 898 epidemiology of coronavirus disease 2019 (COVID-19): disease incidence, daily cumulative 899 index, mortality, and their association with country healthcare resources and economic status. 900 Int J Antimicrob Agents. 55(4), 105946. doi: 10.1016/j.ijantimicag.2020.105946. 
901 [60] Safavi, P.E., Rahimian, K., Doustmohammadi, A., Safari dastjerdei, M., Rasouli, A.,

902 Zahiri, J. 2021. A prediction model for COVID-19 prevalence based on demographic and

903 healthcare parameters in Iran. https://doi.org/10.1101/2021.01.27.21250551

904 [61] Payam, S., Bordbar, N., Ghanbarzadegan, A., Najibi, M., Bastani. P. 2020. Ranking of 905 Iranian Provinces Based on Healthcare Infrastructures: Before and after Implementation of 906 Health Transformation Plan. Cost Effectiveness and Resource Allocation 18(1).

907 [63] Nie, P., Ding, L., Chen, Z. 2021. Income-related health inequality among Chinese adults 908 during the COVID-19 pandemic: evidence based on an online survey. Int J Equity Health 20, 909 106. https://doi.org/10.1186/s12939-021-01448-9

910 [63] Buja, A., Paganini, M., Cocchio, S., Scioni, M., Rebba, V., Baldo, V. 2020. Demographic 911 and socio-economic factors, and healthcare resource indicators associated with the rapid spread 912 of COVID-19 in Northern Italy: An ecological study. PLoS ONE. 15(12), 913 https://doi.org/10.1371/journal.pone.0244535

914 [64] National Institute of Statistics (ISTAT). Main database. [cited 2020 April 13]. Available 915 from: http://dati.istat.it/Index.aspx

916 [65] National Institute of Statistics (ISTAT). Health for All database. [cited 2020 April 13]. 917 Available from: https://www.istat.it/it/archivio/14562.

918 [66] Bizri, A.R., Khachfe, H.H., Fares, M.Y., Musharrafieh, U. 2021. COVID-19 Pandemic: 919 An Insult Over Injury for Lebanon. J Community Health. 46(3), 487-493.

920 [67] Sydow, J., Windeler, A., Muller-Seitz, G. 2012. Path Constitution Analysis: A 921 Methodology for Understanding Path Dependence and Path Creation. Bus Res, 5, 155-176.

922 [68] Rammal, A., Perrin, E., Vrabie, V., Bertrand, I., Chabbert, B. 2017. Classification of 923 lignocellulosic biomass by weighted-covariance factor fuzzy C-means clustering of mid924 infrared and near-infrared spectra, Journal of Chemometrics. 31(2), 1-10.

925 [69] Everson, R., Fieldsend, J. 2006. Multi-class ROC analysis from a multi-objective 
926 optimization perspective. Pattern Recognition Letters 27(8), 918-927.

927

928

929

930

931

932

933

934

935 Supplementary Information

936 Table 3: Correlation between the degrees of possibility and the predictors

\begin{tabular}{|c|c|c|}
\hline \multicolumn{2}{|c|}{ Pearson Correlation } & Sig (2-tailed) \\
\hline Technology used & $\mathbf{- 0 . 0 5 2}$ & $\mathbf{0 . 4 4 2}$ \\
\hline Special event & $\mathbf{0 . 0 6 1}$ & $\mathbf{0 . 3 6 2}$ \\
\hline Procedure & $\mathbf{0 . 0 2 7}$ & $\mathbf{0 . 6 9}$ \\
\hline Experience & $\mathbf{- 0 . 3 6 3}$ & $\mathbf{0 0 0}$ \\
\hline Density & $\mathbf{- 0 . 2 2}$ & $\mathbf{0 . 0 0 1}$ \\
\hline Family Nb & $\mathbf{0 . 1 5 2}$ & $\mathbf{0 . 0 2 2}$ \\
\hline Temperature & $\mathbf{- 0 . 0 0 9}$ & $\mathbf{0 . 8 9 3}$ \\
\hline Lockdown & $\mathbf{- 0 . 2 3 2}$ & $\mathbf{0 0 0}$ \\
\hline Medical Resources & $\mathbf{- 0 . 2 2 4}$ & $\mathbf{0 . 0 0 1}$ \\
\hline Economy Resources & $\mathbf{- 0 . 0 8 1}$ & $\mathbf{0 . 2 3}$ \\
\hline
\end{tabular}

937

938 